\title{
Tropene Derivatives by Sequential, Palladium-Catalyzed, Intermolecular and Transannular, Intramolecular Hydroamination of Cycloheptatriene
}

\author{
Supporting Information \\ Norio Sakai, André Ridder, and John F. Hartwig \\ Department of Chemistry, P.O. Box 208107, Yale University, New Haven, \\ Connecticut 06520-8107
}

\section{Table of Contents}

Spectral data of novel compounds $\quad$ S2-S9

Experimental Details for crystal structure determination of 3a $\quad$ S10-S17

Experimental Details for crystal structure determination of $\mathbf{4 c} \quad$ S18-S24

Table S11: Data on Optimization of the Hydroamination S25

$\begin{array}{ll}\text { References } & \text { S26 }\end{array}$ 
General. All reactions were performed under a nitrogen atmosphere using a nitrogen-filled glovebox or standard Schlenk techniques. All glassware was oven-dried for approximately $1 \mathrm{~h}$ prior to use. Toluene was purged with nitrogen for $45 \mathrm{~min}$ and dried with a solvent purification system containing a $1 \mathrm{~m}$ column of activated alumina. Amines were purified by distillation from the appropriate drying agents or recrystallization from the appropriate solvent. Ligands and acids were purchased from commercial sources and were used without further purification. Cycloheptatriene was purchased from Aldrich and purified by distillation prior to use. Chromatore $^{\circledR} \mathrm{NH}$ was purchased from Fuji Silysia Chemical Ltd. ${ }^{1} \mathrm{H}$ NMR spectra were measured at $400 \mathrm{MHz}$ using tetramethylsilane as an internal standard. ${ }^{13} \mathrm{C}$ NMR spectra were measured at $100.6 \mathrm{MHz}$ using a center peak of chloroform (at $77.0 \mathrm{ppm}$ ) as an internal standard. GC analyses were performed using a DB-1301 narrow bore column. Elemental analyses were performed by Robertson Microlit Laboratories, Madison, NJ or Atlantic Microlab, Inc., Norcross, GA.

General procedure for the hydroamination of primary aromatic amine with cycloheptatriene. In a glovebox, $\operatorname{Pd}(\mathrm{TFA})_{2}(2 \mathrm{~mol} \%, 0.010-0.020 \mathrm{mmol})$ and Xantphos (4 mol\%, $0.020-0.040 \mathrm{mmol})$, and benzoic acid (10 mol\%, 0.050-0.10 mmol) were weighed directly into a screw capped vial. A stir bar, cycloheptatriene (4.0 eq for an amine, 2.0-4.0 mmol, 207-415 $\mu \mathrm{L}$ ), dodecane $(0.15-0.20 \mathrm{mmol})$ as an internal standard, and toluene $(0.5-1.0 \mathrm{~mL})$ were added successively and the reaction mixture was stirred for 2-3 min during which time the solution turned orange or red. Then an amine $(0.50-1.0 \mathrm{mmol})$ was added and the reaction mixture removed from the glovebox. The reaction mixture was stirred at $110{ }^{\circ} \mathrm{C}$. The reaction was monitored by TLC or GC until a complete consumption of amine. The resulting dark red suspension was adsorbed onto silica or alumina gel and purified by flash chromatography (hexane : ethyl acetate $=1: 9$ to $1: 4$ ).

8-phenyl-8-aza-bicyclo[3.2.1]oct-2-ene

and 2,3,3a,4-tetrahydro-3a-methyl-1H-cyclopenta[b]quinoline (4a). Following the general procedure, aniline $(0.499 \mathrm{mmol}, 46.5 \mathrm{mg})$, cycloheptatriene $(2.00 \mathrm{mmol}, 207 \mu \mathrm{L})$, and benzoic acid $(0.100 \mathrm{mmol}, 12.2 \mathrm{mg})$ in toluene $(0.50 \mathrm{~mL})$ yielded $74 \mathrm{mg}(80 \%)$ of $\mathbf{3 a}$ as a pale yellow solid and $11 \mathrm{mg}(12 \%)$ of $\mathbf{4 a}$ as pale yellow oil. 3a: ${ }^{1} \mathrm{H} \mathrm{NMR}\left(\mathrm{CDCl}_{3}\right) \delta 7.13(\mathrm{~d}, 2 \mathrm{H}, J=7.9 \mathrm{~Hz})$, 
$6.80(\mathrm{~d}, 2 \mathrm{H}, J=7.9 \mathrm{~Hz}), 6.67(\mathrm{t}, 1 \mathrm{H}, J=7.9 \mathrm{~Hz}), 6.00(\mathrm{~m}, 1 \mathrm{H}), 5.32(\mathrm{~m}, 1 \mathrm{H}), 4.23(\mathrm{~m}, 1 \mathrm{H}), 4.08$ (s, 1H), $2.56(\mathrm{~m}, 1 \mathrm{H}), 2.24(\mathrm{~m}, 1 \mathrm{H}), 2.04-1.90(\mathrm{~m}, 2 \mathrm{H}), 1.79-1.71(\mathrm{~m}, 1 \mathrm{H}), 1.64-1.57(\mathrm{~m}, 1 \mathrm{H})$; ${ }^{13} \mathrm{C} \mathrm{NMR}\left(\mathrm{CDCl}_{3}\right) \delta 148.3,132.9,129.6,124.7,118.6,117.0,54.8,54.3,35.3,32.0,30.8$; GCMS (m/z) 185, 156; Anal. Calcd. for $\mathrm{C}_{13} \mathrm{H}_{15} \mathrm{~N}$ : C, 84.28; H, 8.16; N, 7.56; Found: C, 84.14; H, 8.10; N, 7.66; 4a: ${ }^{1} \mathrm{H}$ NMR $\left(\mathrm{CDCl}_{3}\right) \delta 6.58(\mathrm{t}, 1 \mathrm{H}, J=8.0 \mathrm{~Hz}), 6.81(\mathrm{~m}, 1 \mathrm{H}), 6.56(\mathrm{~m}, 1 \mathrm{H}), 6.41(\mathrm{~d}, 1 \mathrm{H}$, $J=8.0 \mathrm{~Hz}), 6.05(\mathrm{~s}, 1 \mathrm{H}), 3.89$ (brs, 1H, NH), $2.43(\mathrm{~m}, 1 \mathrm{H}), 1.94-1.84(\mathrm{~m}, 2 \mathrm{H}), 1.79-1.61(\mathrm{~m}$, 2H), $1.08(\mathrm{~s}, 3 \mathrm{H}) ;{ }^{13} \mathrm{C} \mathrm{NMR}\left(\mathrm{CDCl}_{3}\right) \delta 145.5,142.9,127.9,126.7,123.3,118.8,117.1,114.2$, 60.3, 42.2, 29.1, 25.7, 22.4; GCMS (m/z) 185, 170; Anal. Calcd. for $\mathrm{C}_{13} \mathrm{H}_{15} \mathrm{~N}$ : C, 84.28; H, 8.16; N, 7.56; Found: C, 83.89; H, 8.03; N, 7.75.

\section{8-(4-fluorophenyl)-8-aza-bicyclo[3.2.1] oct-2-ene}

(3b)

and

7-fluoro-2,3,3a,4-tetrahydro-3a-methyl-1H-cyclopenta[b]quinoline (4b). General procedure as above with 4-fluoromethylaniline $(0.500 \mathrm{mmol}, 55.6 \mathrm{mg})$, triene $(2.00 \mathrm{mmol}, 415 \mu \mathrm{L})$, benzoic acid $(0.050 \mathrm{mmol}, 6.1 \mathrm{mg})$ in toluene $(0.5 \mathrm{~mL})$ gave $65 \mathrm{mg}(64 \%)$ of $\mathbf{3 b}$ as a pale yellow solid and $25 \mathrm{mg}(25 \%)$ of $\mathbf{4 b}$ as yellow oil. $\mathbf{3 b}$ : ${ }^{1} \mathrm{H}$ NMR $\left(\mathrm{CDCl}_{3}\right) \delta 6.92-6.88(\mathrm{~m}, 2 \mathrm{H}), 6.81-6.78$ $(\mathrm{m}, 2 \mathrm{H}), 6.05(\mathrm{~m}, 1 \mathrm{H}), 5.40(\mathrm{~m}, 1 \mathrm{H}), 4.21(\mathrm{~m}, 1 \mathrm{H}), 4.05(\mathrm{t}, 1 \mathrm{H}, J=5.2 \mathrm{~Hz}), 2.55(\mathrm{dd}, 1 \mathrm{H}, J=$ 22.0, $2.0 \mathrm{~Hz}), 2.10-1.97(\mathrm{~m}, 2 \mathrm{H}), 1.85-1.80(\mathrm{~m}, 1 \mathrm{H}), 1.79-1.69(\mathrm{~m}, 1 \mathrm{H}), 1.67(\mathrm{dd}, 1 \mathrm{H}, J=18.0$, $4.4 \mathrm{~Hz}) ;{ }^{13} \mathrm{C}$ NMR $\left(\mathrm{CDCl}_{3}\right) \delta 156.5\left(\mathrm{~d}, J_{\mathrm{C}, \mathrm{F}}=236 \mathrm{~Hz}\right), 144.7,132.6,124.7,118.0,116.0,55.5$, 54.8, 35.4, 31.8, 30.9; GCMS (m/z) 203; Anal. Calcd. for $\mathrm{C}_{13} \mathrm{H}_{14}$ FNO: C, 76.82; H, 6.94; N, 6.89; Found for C, 76.72; H, 7.07; N, 6.87, 4b: ${ }^{1} \mathrm{H}$ NMR $\left(\mathrm{CDCl}_{3}\right) \delta 6.61(\mathrm{~d}, 2 \mathrm{H}, J=8.4 \mathrm{~Hz}), 6.41(\mathrm{dd}$, $1 \mathrm{H}, J=8.4,4.4 \mathrm{~Hz}), 6.06$ (s, $1 \mathrm{H}), 3.90$ (brs, $1 \mathrm{H}, \mathrm{NH}), 2.52-2.48(\mathrm{~m}, 2 \mathrm{H}), 1.93-1.76$ (m, 4H), $1.13(\mathrm{~s}, 3 \mathrm{H}) ;{ }^{13} \mathrm{C}$ NMR $\left(\mathrm{CDCl}_{3}\right) \delta 156.9\left(\mathrm{~d}, J_{\mathrm{C}, \mathrm{F}}=235 \mathrm{~Hz}\right), 147.7,139.0\left(\mathrm{~d}, J_{\mathrm{C}, \mathrm{F}}=2.3 \mathrm{~Hz}\right), 124.5$ $\left(\mathrm{d}, J_{\mathrm{C}, \mathrm{F}}=7.4 \mathrm{~Hz}\right), 116.5\left(\mathrm{~d}, J_{\mathrm{C}, \mathrm{F}}=2.3 \mathrm{~Hz}\right), 114.5\left(\mathrm{~d}, J_{\mathrm{C}, \mathrm{F}}=7.4 \mathrm{~Hz}\right), 113.8\left(\mathrm{~d}, J_{\mathrm{C}, \mathrm{F}}=23.0 \mathrm{~Hz}\right)$, $112.9\left(\mathrm{~d}, J_{\mathrm{C}, \mathrm{F}}=23.0 \mathrm{~Hz}\right), 60.6,42.1,29.2,25.2,22.5$; GCMS $(\mathrm{m} / \mathrm{z})$ 203; Anal. Calcd. for $\mathrm{C}_{13} \mathrm{H}_{14} \mathrm{FNO}$ : C, 76.82; H, 6.94; N, 6.89; Found for C, 76.85; H, 7.01; N, 6.84.

\section{8-(4-cyanophenyl)-8-aza-bicyclo[3.2.1]oct-2-ene}

(3c)

and 8-cyano-2,3,3a,4-tetrahydro-3a-methyl-1H-cyclopenta[b]quinoline (4c). General procedure as above with 4-aminobenzonitrile $(0.500 \mathrm{mmol}, 59.2 \mathrm{mg})$, triene $(2.00 \mathrm{mmol}, 207 \mu \mathrm{L})$, benzoic acid $(0.050 \mathrm{mmol}, 6.1 \mathrm{mg})$ in toluene $(0.5 \mathrm{~mL})$ gave $69 \mathrm{mg}(66 \%)$ of $\mathbf{3 c}$ as brown solid and 32 $\mathrm{mg}(24 \%)$ of $\mathbf{4 c}$ as a bright yellow crystal. $3 \mathrm{c}:{ }^{1} \mathrm{H} \mathrm{NMR}\left(\mathrm{CDCl}_{3}\right) \delta 7.37(\mathrm{~d}, 2 \mathrm{H}, J=8.8 \mathrm{~Hz}), 6.78$ 
$(\mathrm{d}, 2 \mathrm{H}, J=8.5 \mathrm{~Hz}), 6.01(\mathrm{~m}, 1 \mathrm{H}), 5.35(\mathrm{~m}, 1 \mathrm{H}), 4.28(\mathrm{~m}, 1 \mathrm{H}), 4.15(\mathrm{t}, 1 \mathrm{H}, J=5.1 \mathrm{~Hz}), 2.56(\mathrm{~m}$, $1 \mathrm{H}), 2.25(\mathrm{~m}, 1 \mathrm{H}), 2.04-1.60(\mathrm{~m}, 4 \mathrm{H}) ;{ }^{13} \mathrm{C} \mathrm{NMR}\left(\mathrm{CDCl}_{3}\right) \delta 151.1,133.8,132.5,124.7,120.8$, 115.7, 99.3, 54.4, 54.2, 34.9, 32.3, 30.3; GCMS (m/z) 210; Anal. Calcd. for $\mathrm{C}_{14} \mathrm{H}_{14} \mathrm{~N}_{2}$ : C, 79.97; $\mathrm{H}, 6.71$; N, 13.32; Found for C, 79.85; H, 6.73; N, 13.20, 4c: ${ }^{1} \mathrm{H}$ NMR $\left(\mathrm{CDCl}_{3}\right) \delta 7.10(\mathrm{~d}, 1 \mathrm{H}, J=$ $7.9 \mathrm{~Hz}), 7.02(\mathrm{~d}, 1 \mathrm{H}, J=7.9 \mathrm{~Hz}), 6.38$ (s, 1H), 5.99 (s, 1H), 4.51 (brs, 1H, NH), $2.45(\mathrm{~m}, 1 \mathrm{H})$, 1.95-1.82 (m, 2H), 1.79-1.64 (m, 2H), $1.12(\mathrm{~s}, 3 \mathrm{H}) ;{ }^{13} \mathrm{C} \mathrm{NMR}\left(\mathrm{CDCl}_{3}\right) \delta 146.5,146.3,132.1$, 130.0, 122.6, 121.0, 115.6, 113.5, 99.6, 60.4, 41.5, 28.6, 26.6, 21.3; GCMS (m/z) 210, 195; Anal. Calcd. for $\mathrm{C}_{14} \mathrm{H}_{14} \mathrm{~N}_{2}$ : C, 79.97; H, 6.71; N, 13.32; Found for C, 79.92; H, 6.73; N, 13.24.

Ethyl 4-(8-aza-bicyclo[3-2.1]oct-2-en-8-yl)benzoate

(3d)

and 7-carboethoxy-2,3,3a,4-tetrahydro-3a-methyl-1H-cyclopenta[b]quinoline $\quad(4 d)$ General procedure as above with 4-aminoethyl benzoate $(1.000 \mathrm{mmol}, 165.2 \mathrm{mg})$, triene (4.00 mmol, 415 $\mu \mathrm{L})$, benzoic acid $(0.100 \mathrm{mmol}, 12.2 \mathrm{mg})$ in toluene $(1 \mathrm{~mL})$ gave $145 \mathrm{mg}(56 \%)$ of $3 \mathbf{d}$ as pale yellow oil and $83 \mathrm{mg}(32 \%)$ of $\mathbf{4 d}$ as a white solid. $3 \mathbf{d}:{ }^{1} \mathrm{H} \mathrm{NMR}\left(\mathrm{CDCl}_{3}\right) \delta 7.92(\mathrm{dd}, 2 \mathrm{H}, J=9.6$, $2.5 \mathrm{~Hz}), 6.82(\mathrm{~d}, 2 \mathrm{H}, J=9.6,2.5 \mathrm{~Hz}), 6.06(\mathrm{~m}, 1 \mathrm{H}), 5.40(\mathrm{~m}, 1 \mathrm{H}), 4.26(\mathrm{~m}, 1 \mathrm{H}), 4.22(\mathrm{q}, 2 \mathrm{H}, J=$ $7.1 \mathrm{~Hz}), 4,14(\mathrm{t}, 1 \mathrm{H}, J=5.0 \mathrm{~Hz}), 2.57(\mathrm{~m}, 1 \mathrm{H}), 2.22(\mathrm{~m}, 1 \mathrm{H}), 2.05-1.58(\mathrm{~m}, 4 \mathrm{H}), 1.26(\mathrm{t}, 3 \mathrm{H}, J=$ $6.8 \mathrm{~Hz}) ;{ }^{13} \mathrm{C} \mathrm{NMR}\left(\mathrm{CDCl}_{3}\right) \delta 167.4,152.0,132.9,131.7,124.8,119.5,115.3,60.7,54.6,54.4$, 35.2, 32.4, 30.6, 15.0; GCMS (m/z) 257, 214; Anal. Calcd. for $\mathrm{C}_{16} \mathrm{H}_{19} \mathrm{NO}_{2}: \mathrm{C}, 74.68 ; \mathrm{H}, 7.44 ; \mathrm{N}$, 5.44; Found for C, 74.66; H, 7.77; N, 5.30, 4d: ${ }^{1} \mathrm{H}$ NMR $\left(\mathrm{CDCl}_{3}\right) \delta 7.64(\mathrm{~d}, 1 \mathrm{H}, J=8.1 \mathrm{~Hz}), 7.55$ $(\mathrm{s}, 1 \mathrm{H}), 6.43(\mathrm{~d}, 1 \mathrm{H}, J=8.1 \mathrm{~Hz}), 4.31(\mathrm{brs}, 1 \mathrm{H}), 4.27(\mathrm{q}, 2 \mathrm{H}, J=7.2 \mathrm{~Hz}), 2.51(\mathrm{td}, 2 \mathrm{H}, J=8.1$ $\mathrm{Hz}, J=2.0 \mathrm{~Hz}), 1.9-1.7(\mathrm{~m}, 4 \mathrm{H}), 1.33(\mathrm{t}, 3 \mathrm{H}, J=7.2 \mathrm{~Hz}), 1.16(\mathrm{~s}, 3 \mathrm{H}) ;{ }^{13} \mathrm{C} \mathrm{NMR}\left(\mathrm{CDCl}_{3}\right) \delta 167.6$, $147.1,145.4$, 130.3, 128.4, 122.1, 120.2, 116.8, 113.2, 60.8, 60.7, 41.9, 28.9, 26.6, 21.9, 15.1; GCMS (m/z) 257, 242, 214; Anal. Calcd. for $\mathrm{C}_{16} \mathrm{H}_{19} \mathrm{NO}_{2}$ : C, 74.68; H, 7.44; N, 5.44; Found for C, 74.41; H, 7.39; N, 5.18.

8-[(1,1'-biphenyl)-4-yl]-8-aza-bicyclo[3.2.1]oct-2-ene (3e) and 7-phenyl-2,3,3a,4-tetrahydro-3a-methyl-1H-cyclopenta[b]quinoline (4e). General procedure as above with 4-aminobiphenyl $(0.500 \mathrm{mmol}, 86.3 \mathrm{mg})$, triene $(2.00 \mathrm{mmol}, 207 \mu \mathrm{L})$, benzoic acid $(0.050 \mathrm{mmol}, 6.1 \mathrm{mg})$ in toluene $(0.5 \mathrm{~mL})$ gave $56 \mathrm{mg}(42 \%)$ of $\mathbf{3 e}$ as a yellow solid and 51 mg $(38 \%)$ of $4 \mathbf{e}$ as red oil. 3e: ${ }^{1} \mathrm{H} \operatorname{NMR}\left(\mathrm{CDCl}_{3}\right) \delta 7.45(\mathrm{~d}, 2 \mathrm{H}, J=8.8 \mathrm{~Hz}), 7.37(\mathrm{~d}, 2 \mathrm{H}, J=8.8$ $\mathrm{Hz}), 7.27(\mathrm{t}, 2 \mathrm{H}, J=7.8 \mathrm{~Hz}), 7.14(\mathrm{t}, 2 \mathrm{H}, J=7.8 \mathrm{~Hz}), 6.82(\mathrm{~d}, 2 \mathrm{H}, J=8.8 \mathrm{~Hz}), 5.98(\mathrm{~m}, 1 \mathrm{H})$, 
$5.31(\mathrm{~m}, 1 \mathrm{H}), 4.21(\mathrm{~m}, 1 \mathrm{H}), 4.07(\mathrm{t}, 1 \mathrm{H}, J=5.1 \mathrm{~Hz}), 2.78(\mathrm{~m}, 1 \mathrm{H}), 2.22(\mathrm{~m}, 1 \mathrm{H}), 2.06-1.86(\mathrm{~m}$, 2H), $1.72(\mathrm{~m}, 1 \mathrm{H}), 1.58(\mathrm{~m}, 1 \mathrm{H}) ;{ }^{13} \mathrm{C} \mathrm{NMR}\left(\mathrm{CDCl}_{3}\right) \delta 147.6,141.8,133.0,131.2,129.2,128.2$, 126.9, 126.7, 124.8, 117.1, 54.9, 54.4, 35.3, 32.1, 30.8; GCMS (m/z) 261, 184; Anal. Calcd. for $\mathrm{C}_{19} \mathrm{H}_{19} \mathrm{~N}$ : C, 87.31; H, 7.33; N, 5.36; Found for C, 87.15; H, 7.39; N, 5.15, 4e: ${ }^{1} \mathrm{H}$ NMR $\left(\mathrm{CDCl}_{3}\right)$ 87.48-7.37 (m, 2H), 7.32-7.27 (m, 3H), 7.17-7.11 (m, 3H), 6.12 (s, 1H), 2.54 (brs, 1H), 1.98-1.77 $(\mathrm{m}, 5 \mathrm{H}), 1.19(\mathrm{~s}, 3 \mathrm{H}) ;{ }^{13} \mathrm{C} \mathrm{NMR}\left(\mathrm{CDCl}_{3}\right) \delta 145.9,142.5,142.0,133.0,129.2,128.2,126.9,126.6$, 125.3, 124.6, 116.9, 114.2, 60.5, 42.2, 29.1, 25.9, 22.3; GCMS (m/z) 261, 246, 184; Anal. Calcd. for $\mathrm{C}_{19} \mathrm{H}_{19} \mathrm{~N}$ : C, 87.31; H, 7.33; N, 5.36; Found for C, 87.01; H, 7.21; N, 5.26.

\section{8-(4-methoxyphenyl)-8-aza-bicyclo[3.2.1]oct-2-ene}

and

7-methoxy-2,3,3a,4-tetrahydro-3a-methyl-1H-cyclopenta[b]quinoline (4f). General procedure as above with 4-methoxyaniline $(0.500 \mathrm{mmol}, 61.5 \mathrm{mg})$, triene $(2.00 \mathrm{mmol}, 207 \mu \mathrm{L})$, benzoic acid $(0.050 \mathrm{mmol}, 6.1 \mathrm{mg})$ in toluene $(1 \mathrm{~mL})$ gave $85 \mathrm{mg}(73 \%)$ of $\mathbf{3 f}$ as a pale yellow solid and $10 \mathrm{mg}(9 \%)$ of $\mathbf{4 f}$ as pale yellow oil. 3f: ${ }^{1} \mathrm{H} \mathrm{NMR}\left(\mathrm{CDCl}_{3}\right) \delta 6.81(\mathrm{~d}, 2 \mathrm{H}, J=9.9 \mathrm{~Hz}), 6.77(\mathrm{~d}, 2 \mathrm{H}$, $J=9.9 \mathrm{~Hz}), 6.05(\mathrm{~m}, 1 \mathrm{H}), 5.39(\mathrm{~m}, 1 \mathrm{H}), 4.19(\mathrm{~m}, 1 \mathrm{H}), 4.03(\mathrm{~m}, 1 \mathrm{H}), 3.72(\mathrm{~s}, 3 \mathrm{H}), 2.54(\mathrm{~d}, 1 \mathrm{H}, J$ $=18.0 \mathrm{~Hz}), 2.28-2.3(\mathrm{~m}, 1 \mathrm{H}), 2.04-2.08(\mathrm{~m}, 1 \mathrm{H}), 1.95-2.00(\mathrm{~m}, 1 \mathrm{H}), 1.78-1.81(\mathrm{~m}, 1 \mathrm{H}), 1.64(\mathrm{~d}$, $1 \mathrm{H}, J=18.0 \mathrm{~Hz}) ;{ }^{13} \mathrm{C} \mathrm{NMR}\left(\mathrm{CDCl}_{3}\right) \delta 152.9,142.2,132.0,123.9,117.6,114.3,55.5,54.9,54.2$, 34.6, 31.1, 30.1; GCMS (m/z) 215, 186; Anal. Calcd. for $\mathrm{C}_{14} \mathrm{H}_{17} \mathrm{NO}$ : C, 78.10; H, 7.96; N, 6.51; Found for C, 78.19; H, 7.85; N, 6.43, 4f: ${ }^{1} \mathrm{H}$ NMR $\left(\mathrm{CDCl}_{3}\right) \delta 6.50-6.41(\mathrm{~m}, 2 \mathrm{H}), 6.02(\mathrm{~s}, 1 \mathrm{H})$, $3.68(\mathrm{~s}, 3 \mathrm{H}), 2.43(\mathrm{~m}, 2 \mathrm{H}), 1.86-1.68(\mathrm{~m}, 4 \mathrm{H}), 1.06(\mathrm{~s}, 3 \mathrm{H}) ;{ }^{13} \mathrm{C} \mathrm{NMR}\left(\mathrm{CDCl}_{3}\right)$ 8153.2, 147.2, 136.9, 124.5, 117.0, 114.9, 113.3, 112.3, 60.5, 56.1, 42.1, 30.8, 25.0, 22.6; GCMS (m/z) 215, 200; Anal. Calcd. for $\mathrm{C}_{14} \mathrm{H}_{17} \mathrm{NO}$ : C, 78.10; H, 7.96; N, 6.51; Found for C, 78.36; H, 7.82; N, 6.15 .

8-(4-methylphenyl)-8-aza-bicyclo[3.2.1]oct-2-ene

(3g)

and 7-methyl-2,3,3a,4-tetrahydro-3a-methyl-1H-cyclopenta[b]quinoline (4g). General procedure as above with $p$-toluidine $(0.500 \mathrm{mmol}, 53.5 \mathrm{mg})$, triene $(2.00 \mathrm{mmol}, 207 \mu \mathrm{L})$, benzoic acid $(0.050 \mathrm{mmol}, 6.1 \mathrm{mg})$ in toluene $(0.5 \mathrm{~mL})$ gave $79 \mathrm{mg}(80 \%)$ of $\mathbf{3 g}$ as a pale yellow solid and 29 mg $(15 \%)$ of $\mathbf{4 g}$ as pale yellow oil. $3 \mathrm{~g}:{ }^{1} \mathrm{H} \mathrm{NMR}\left(\mathrm{CDCl}_{3}\right) \delta 7.02(\mathrm{~d}, 2 \mathrm{H}, J=8.0 \mathrm{~Hz}), 6.79(\mathrm{~d}, 2 \mathrm{H}$, $J=8.0 \mathrm{~Hz}), 6.07(\mathrm{~m}, 1 \mathrm{H}), 5.41(\mathrm{~m}, 1 \mathrm{H}), 4.26(\mathrm{~m}, 1 \mathrm{H}), 4.11(\mathrm{t}, 1 \mathrm{H}, J=5.2 \mathrm{~Hz}), 2.62(\mathrm{~d}, 1 \mathrm{H}, J$ $=18.0 \mathrm{~Hz}), 2.3-2.33(\mathrm{~m}, 1 \mathrm{H}), 2.24(\mathrm{~s}, 3 \mathrm{H}), 2.07-2.10(\mathrm{~m}, 1 \mathrm{H}), 1.99-2.02(\mathrm{~m}, 1 \mathrm{H}), 1.81-1.85(\mathrm{~m}$, 
$1 \mathrm{H}), 1.65(\mathrm{~d}, 1 \mathrm{H}, J=18.0 \mathrm{~Hz}) ;{ }^{13} \mathrm{C} \mathrm{NMR}\left(\mathrm{CDCl}_{3}\right) \delta 146.0,133.0,130.1,127.7,124.6,117.0,54.9$, 54.4, 35.2, 31.8, 30.8, 21.0; GCMS (m/z) 199; Anal. Calcd. for $\mathrm{C}_{14} \mathrm{H}_{17} \mathrm{~N}$ : C, 84.37; H, 8.60; N, 7.03; Found for C, 83.99; H, 8.97; N, 6.95, 4g: ${ }^{1} \mathrm{H}$ NMR $\left(\mathrm{CDCl}_{3}\right) \delta 6.75(\mathrm{~d}, 1 \mathrm{H}, J=7.6 \mathrm{~Hz}), 6.72$ (s, 1H), 6.42 (d, 1H, J = 7.6 Hz), 6.09 (s, 1H), 3.80 (brs, 1H, NH), 2.49-2.53 (m, 2H), 2.21 (s, 3H), 1.73-1.97 (m, 4H), $1.18(\mathrm{~s}, 9 \mathrm{H}), 1.15(\mathrm{~s}, 3 \mathrm{H}) ;{ }^{13} \mathrm{C}$ NMR $\left(\mathrm{CDCl}_{3}\right) \delta 145.8,140.6,128.3,127.8$ 127.2, 123.3, 117.1, 114.1, 60.3, 42.2, 29.1, 25.4, 22.5, 21.1; GCMS (m/z) 199; Anal. Calcd. for $\mathrm{C}_{14} \mathrm{H}_{17} \mathrm{~N}$ : C, 84.37; H, 8.60; N, 7.03; Found for C, 84.25; H, 8.84; N, 6.87.

8-(3,5-Bis(trifluoromethyl)phenyl)-8-aza-bicyclo[3.2.1]oct-2-ene (3h). General procedure as above with 3,5-bis(trifluoromethyl)aniline (1.000 mmol, $229.1 \mathrm{mg})$, triene (4.00 mmol, $415 \mu \mathrm{L})$, benzoic acid $(0.100 \mathrm{mmol}, 12.2 \mathrm{mg})$ in toluene $(1 \mathrm{~mL})$ gave $197 \mathrm{mg}(61 \%)$ of $\mathbf{3 h}$ as a white solid. 3h: ${ }^{1} \mathrm{H}$ NMR $\left(\mathrm{CDCl}_{3}\right) \delta 7.10$ (s, 3H), $6.02(\mathrm{~m}, 1 \mathrm{H}), 5.34(\mathrm{~m}, 1 \mathrm{H}), 4.27(\mathrm{~m}, 1 \mathrm{H}), 4.11(\mathrm{t}, 1 \mathrm{H}, J=$ $5.4 \mathrm{~Hz}), 2.51(\mathrm{~m}, 1 \mathrm{H}), 2.27(\mathrm{~m}, 1 \mathrm{H}), 2.08-1.67(\mathrm{~m}, 4 \mathrm{H}) ;{ }^{13} \mathrm{C} \mathrm{NMR}\left(\mathrm{CDCl}_{3}\right)$ 8149.0, 132.9, 132.6, 132.3, 125.1, 116.0, 111.3, 55.2, 54.7, 35.3, 32.5, 30.7; GCMS (m/z) 321; Anal. Calcd. for $\mathrm{C}_{15} \mathrm{H}_{13} \mathrm{NF}_{6}$ : C, 56.08; H, 4.08; N, 4.36; Found for C, 56.19; H, 3.87; N, 4.03.

8-(3,4,5-Tris(methoxy)phenyl)-8-aza-bicyclo[3.2.1]oct-2-ene (3i). General procedure as above with 3,4,5-tris(trimethoxy)aniline $(0.500 \mathrm{mmol}, 91.6 \mathrm{mg})$, triene $(2.00 \mathrm{mmol}, 207 \mu \mathrm{L})$, benzoic acid $(0.050 \mathrm{mmol}, 6.1 \mathrm{mg})$ in toluene $(0.5 \mathrm{~mL})$ gave $122 \mathrm{mg}(90 \%)$ of 3i as colourless oil. $3 \mathbf{i}:{ }^{1} \mathrm{H}$ NMR $\left(\mathrm{CDCl}_{3}\right) \delta 6.05(\mathrm{~m}, 2 \mathrm{H}), 6.03(\mathrm{~m}, 1 \mathrm{H}), 5.45(\mathrm{~m}, 1 \mathrm{H}), 4.21(\mathrm{dd}, 1 \mathrm{H}, J=6.8,4.8 \mathrm{~Hz}), 4.05(\mathrm{t}$, $1 \mathrm{H}, J=5.2 \mathrm{~Hz}), 3.79(\mathrm{~s}, 6 \mathrm{H}), 3.73(\mathrm{~s}, 3 \mathrm{H}), 2.55(\mathrm{dd}, 1 \mathrm{H}, J=18.0,1.6 \mathrm{~Hz}), 2.28(\mathrm{~m}, 1 \mathrm{H})$, 2.02-1.77 (m, 3H), $1.65(\mathrm{dd}, 1 \mathrm{H}, J=18.0,1.6 \mathrm{~Hz}) ;{ }^{13} \mathrm{C} \mathrm{NMR}\left(\mathrm{CDCl}_{3}\right) \delta 154.2,145.0,132.8$, 131.2, 125.0, 111.3, 61.7, 56.6, 55.4, 54.9, 35.4, 32.2, 30.8; GCMS (m/z) 275; Anal. Calcd. for $\mathrm{C}_{16} \mathrm{H}_{21} \mathrm{NO}_{3}: \mathrm{C}, 69.79 ; \mathrm{H}, 7.69 ; \mathrm{N}, 5.09$; Found for C, 69.94; H, 7.99; N, 4.92.

\section{8-(3-quinolinyl)-8-aza-bicyclo[3.2.1]oct-2-ene}

General procedure as above with 3-aminoquinoline $(0.500 \mathrm{mmol}, 72.1 \mathrm{mg})$, triene $(2.00 \mathrm{mmol}$, $207 \mu \mathrm{L})$, benzoic acid $(0.050 \mathrm{mmol}, 6.1 \mathrm{mg})$ in toluene $(0.5 \mathrm{~mL})$ gave $68 \mathrm{mg}(58 \%)$ of $\mathbf{3 j}$ as dark yellow oil and $17 \mathrm{mg}(14 \%)$ of $\mathbf{4 j}$ as dark red oil. 3j: ${ }^{1} \mathrm{H}$ NMR $\left(\mathrm{CDCl}_{3}\right) \delta 8.70(\mathrm{~d}, 1 \mathrm{H}, J=2.4 \mathrm{~Hz})$, $7.89(\mathrm{dd}, 1 \mathrm{H}, J=8.0,2.4 \mathrm{~Hz}), 7.54(\mathrm{dd}, 1 \mathrm{H}, J=8.0,2.4 \mathrm{~Hz}), 7.35(\mathrm{~m}, 2 \mathrm{H}), 7.22(\mathrm{~d}, 1 \mathrm{H}, J=2.4$ 
$\mathrm{Hz}), 6.03(\mathrm{~m}, 1 \mathrm{H}), 5.31(\mathrm{~m}, 1 \mathrm{H}), 4.41(\mathrm{~m}, 1 \mathrm{H}), 4.18(\mathrm{~m}, 1 \mathrm{H}), 2.51(\mathrm{dd}, 1 \mathrm{H}, J=18.0,3.9 \mathrm{~Hz})$, $2.31(\mathrm{~m}, 1 \mathrm{H}), 2.06-2.00(\mathrm{~m}, 2 \mathrm{H}), 1.81-1.83(\mathrm{~m}, 1 \mathrm{H}), 1.71(\mathrm{~d}, 1 \mathrm{H}, J=18.0,3.9 \mathrm{~Hz}) ;{ }^{13} \mathrm{C} \mathrm{NMR}$ $\left(\mathrm{CDCl}_{3}\right) \delta 144.8,142.7,142.0,132.1,129.8,129.4,127.3,126.7,126.1,124.8,118.0,55.3,54.6$, 35.2, 32.6, 30.8; GCMS (m/z) 236, 207; Anal. Calcd. for $\mathrm{C}_{16} \mathrm{H}_{16} \mathrm{~N}_{2}: \mathrm{C}, 81.32 ; \mathrm{H}, 6.82 ; \mathrm{N}, 11.85$; Found for C, 80.96; H, 7.06; N, 11.93, 4j: ${ }^{1} \mathrm{H} \mathrm{NMR}\left(\mathrm{CDCl}_{3}\right) \delta 8.22(\mathrm{~s}, 1 \mathrm{H}), 7.87(\mathrm{dd}, 2 \mathrm{H}, J=8.0$, $1.6 \mathrm{~Hz}), 7.67(\mathrm{dd}, 1 \mathrm{H}, J=8.0,1.6 \mathrm{~Hz}), 7.38(\mathrm{td}, 1 \mathrm{H}, J=7.9,1.4 \mathrm{~Hz}), 7.33(\mathrm{td}, 1 \mathrm{H}, J=7.9,1.4$ Hz), $3.03(\mathrm{dd}, 1 \mathrm{H}, J=18,0,2.4 \mathrm{~Hz}), 2.94(\mathrm{dd}, 1 \mathrm{H}, J=18.0,6.4 \mathrm{~Hz}), 2.11-2.13(\mathrm{~m}, 1 \mathrm{H})$, 1.82-1.75 (m, 4H), $1.65(\mathrm{~m}, 1 \mathrm{H}), 1.39(\mathrm{~m} .1 \mathrm{H}), 1.26(\mathrm{~s}, 3 \mathrm{H}) ;{ }^{13} \mathrm{C} \mathrm{NMR}\left(\mathrm{CDCl}_{3}\right) \delta 142.9,142.2$, 136.8, 130.1, 129.1, 127.0, 124.5, 121.6, 116.5, 58.8, 42.2, 41.1, 30.4, 26.1, 22.8, 20.8; GCMS $(m / z)$ 238, 223, 195; Anal. Calcd. for $\mathrm{C}_{16} \mathrm{H}_{18} \mathrm{~N}_{2}$ : C, 80.63; H, 7.61; N, 11.75; Found for C, 80.53; $\mathrm{H}, 7.84 ; \mathrm{N}, 11.42$.

8-(3-pyridinyl)-8-aza-bicyclo[3.2.1]oct-2-ene

(3k)

and

7,8,8a,9-tetrahydro-8a-methyl-6H-cyclopenta[b][1,7]naphthyridine (4k): General procedure as above with 2-aminopyridine $(0.50 \mathrm{mmol}, 47.1 \mathrm{mg})$, triene $(2.00 \mathrm{mmol}, 207 \mu \mathrm{L})$, TFA $(0.050$ mmol, $4.0 \mu \mathrm{L})$ in toluene $(0.5 \mathrm{~mL})$ gave $43 \mathrm{mg}(41 \%)$ of $3 \mathbf{k}$ as yellow oil and $19 \mathrm{mg}(18 \%)$ of $4 \mathbf{k}$ as dark yellow oil. 3k; ${ }^{1} \mathrm{H} \mathrm{NMR}\left(\mathrm{CDCl}_{3}\right) \delta 8.20(\mathrm{~d}, 1 \mathrm{H}, J=2.8 \mathrm{~Hz}), 7.94(\mathrm{dd}, 1 \mathrm{H}, J=4.4,2.8 \mathrm{~Hz})$, 7.10-7.03 (m, 2H), $6.03(\mathrm{~m}, 1 \mathrm{H}), 5.35(\mathrm{~m}, 1 \mathrm{H}), 4.26(\mathrm{~m}, 1 \mathrm{H}), 4.09(\mathrm{t}, 1 \mathrm{H}, J=4.4 \mathrm{~Hz}), 2.51(\mathrm{td}$, $1 \mathrm{H}, J=15.6,2.4 \mathrm{~Hz}), 2.28(\mathrm{~m}, 1 \mathrm{H}), 2.05-1.96(\mathrm{~m}, 1 \mathrm{H}), 1.80-1.75(\mathrm{~m}, 1 \mathrm{H}), 1.68$ (dd, $J=18.0,4.4$ $\mathrm{Hz}) ;{ }^{13} \mathrm{C} \mathrm{NMR}\left(\mathrm{CDCl}_{3}\right) \delta 144.5,139.7,139.4,132.4,124.7,124.1,123.6,54.8,54.1,35.2,31.9$, 30.7; GCMS (m/z) 186, 157, 78; Anal. Calcd. for $\mathrm{C}_{12} \mathrm{H}_{14} \mathrm{~N}_{2}$ : C, 77.38; H, 7.58; N, 15.04; Found for C, 76.99; H, 7.85; N, 14.85, 4k: ${ }^{1} \mathrm{H}-\mathrm{NMR}\left(\mathrm{CDCl}_{3}\right) \delta 7.82(\mathrm{dd}, 1 \mathrm{H}, J=4.4,1.4 \mathrm{~Hz}), 6.79(\mathrm{dd}$, $1 \mathrm{H}, J=8.0,4.4 \mathrm{~Hz}), 6.71(\mathrm{dd}, 1 \mathrm{H}, J=8.0,1.6 \mathrm{~Hz}), 6.28(\mathrm{~d}, 1 \mathrm{H}, J=1.6 \mathrm{~Hz}), 5.41(\mathrm{~m}, 1 \mathrm{H}), 2.55$ $(\mathrm{m}, 2 \mathrm{H}), 1.81-1.83(\mathrm{~m}, 4 \mathrm{H}), 1.18(\mathrm{~s}, 3 \mathrm{H}) ;{ }^{13} \mathrm{C} \mathrm{NMR}\left(\mathrm{CDCl}_{3}\right) \delta 151.1,143.3,139.8,139.2,122.5$, 120.0, 118.9, 60.6, 42.0, 29.4, 26.5, 22.2; GCMS (m/z) 186, 171, 158; Anal. Calcd. for $\mathrm{C}_{12} \mathrm{H}_{14} \mathrm{~N}_{2}$ : C, 77.38; H, 7.58; N, 15.04; Found for C, 77.34; H, 7.59; N, 14.79.

General procedure for the hydroamination of primary alkyl amines with cycloheptatriene. In a glovebox, $\operatorname{Pd}(\mathrm{TFA})_{2}(5 \mathrm{~mol} \%, 0.025-0.050 \mathrm{mmol})$ and Xantphos (7 mol\%, 0.35-0.70 mmol) were weighed directly into a screw capped vial. A stir bar, cycloheptatriene (4.0 eq for an amine, 2.00-4.00 mmol, 207-415 $\mu \mathrm{L})$, dodecane $(0.15-0.20 \mathrm{mmol})$ as an internal standard, and toluene 
(0.5-1.0 mL) were added successively and the reaction mixture was stirred for 2-3 min during which time the solution turned dark red. Then an amine $(0.50-1.00 \mathrm{mmol})$ was added and the vial was sealed with a cap containing a PTFE septum and removed from the glovebox. To the resulting solution was added trifluoroacetic acid (20 mol\%, 0.10-0.20 mmol) via syringe through the septum. The reaction mixture was heated at $110^{\circ} \mathrm{C}$. The reaction was monitored by TLC or GC until a complete consumption of amine. The resulting dark red suspension was adsorbed onto alumina or Chromatorex ${ }^{\circledR} \mathrm{NH}$ and purified by flash chromatography (hexane : ethyl acetate $=9: 1$ to $1: 9)$.

8-phenylmethyl-8-aza-bicyclo[3.2.1 $]$ oct-2-ene $\quad(\mathbf{6 a}) .{ }^{1}$ General procedure as above with benzylamine $(0.500 \mathrm{mmol}, 53.6 \mathrm{mg})$, triene $(2.00 \mathrm{mmol}, 207 \mu \mathrm{L})$ in toluene $(0.5 \mathrm{~mL})$ gave 68 mg $(68 \%)$ of 6a as yellow oil. 6a: ${ }^{1} \mathrm{H}$ NMR $\left(\mathrm{CDCl}_{3}\right) \delta 7.43-7.40(\mathrm{~m}, 2 \mathrm{H}), 7.36-7.32(\mathrm{~m}, 2 \mathrm{H})$, 7.29-7.25 (m, 1H), $5.80(\mathrm{~m}, 1 \mathrm{H}), 5.67(\mathrm{~m}, 1 \mathrm{H}), 3.75(\mathrm{dd}, 2 \mathrm{H}, J=16.4,13.4 \mathrm{~Hz}), 3.31(\mathrm{dd}, 1 \mathrm{H}, J$ $=4.8,1.2 \mathrm{~Hz}), 3.25(\mathrm{t}, 1 \mathrm{H}, J=4.8 \mathrm{~Hz}), 2.54(\mathrm{dd}, 1 \mathrm{H}, J=18.0,2.4 \mathrm{~Hz}), 2.25-2.20(\mathrm{~m}, 1 \mathrm{H})$, 2.05-2.00 (m, 1H), $1.88(\mathrm{td}, 1 \mathrm{H}, J=9.6,2.8 \mathrm{~Hz}), 1.67(\mathrm{dd}, 1 \mathrm{H}, J=18.0,2.4 \mathrm{~Hz}) ;{ }^{13} \mathrm{C} \mathrm{NMR}$ $\left(\mathrm{CDCl}_{3}\right) \delta 140.5,131.5,129.3,128.7,127.3,124.1,57.1,56.4,53.7,34.8,32.4$, 30.6: GCMS $(m / z) 199,170,91$.

8-(4-methoxyphenyl)methyl)-8-aza-bicyclo[3.2.1]oct-2-ene (6b). General procedure as above with 4-methoxybenzylamine (1.000 mmol, $137.3 \mathrm{mg})$, triene $(4.00 \mathrm{mmol}, 415 \mu \mathrm{L})$ in toluene $(1$ $\mathrm{mL})$ gave $160 \mathrm{mg}(70 \%)$ of $\mathbf{6 b}$ as yellow oil. $\mathbf{6 b}:{ }^{1} \mathrm{H} \mathrm{NMR}\left(\mathrm{CDCl}_{3}\right) \delta 7.27(\mathrm{~d}, 2 \mathrm{H}, J=9.2 \mathrm{~Hz})$, $6.81(\mathrm{~d}, 2 \mathrm{H}, J=9.2 \mathrm{H}), 5.74,(\mathrm{~m}, 1 \mathrm{H}), 5.61(\mathrm{~m}, 1 \mathrm{H}), 3.76(\mathrm{~s}, 3 \mathrm{H}), 3.62(\mathrm{dd}, 2 \mathrm{H}, J=15.4,13.0$ $\mathrm{Hz}), 3.24(\mathrm{dd}, 1 \mathrm{H}, J=7.2,4.8 \mathrm{~Hz}), 3.18(\mathrm{t}, 1 \mathrm{H}, J=5.6 \mathrm{~Hz}), 2.50(\mathrm{dd}, 1 \mathrm{H}, J=17.6,2.0 \mathrm{~Hz}), 2.15$ $(\mathrm{m}, 1 \mathrm{H}), 1.96(\mathrm{~m}, 1 \mathrm{H}), 1.82(\mathrm{td}, 1 \mathrm{H}, J=7.2,2.8 \mathrm{~Hz}), 1.63-1.55(\mathrm{~m}, 1 \mathrm{H}) ;{ }^{13} \mathrm{C} \mathrm{NMR}\left(\mathrm{CDCl}_{3}\right)$ \$159.0, 132.7, 131.5, 130.4, 124.2, 114.1, 56.9, 56.2, 55.8, 53.1, 34.7, 32.4, 30.6; GCMS (m/z) 229, 198; Anal. Calcd. for $\mathrm{C}_{15} \mathrm{H}_{19} \mathrm{NO}$ : C, 78.56; H, 8.35; N, 6.11; Found for C, 78.45; H, 8.60; N, 5.90 .

8-(4-trifluorophenyl)methyl)-8-aza-bicyclo[3.2.1]oct-2-ene (6c). General procedure as above with 4-trifluoromethylbenzylamine $(0.500 \mathrm{mmol}, 87.6 \mathrm{mg})$, triene $(2.00 \mathrm{mmol}, 207 \mu \mathrm{L})$ in toluene $(0.5 \mathrm{~mL})$ gave $86 \mathrm{mg}(62 \%)$ of $\mathbf{6 c}$ as yellow oil. $6 \mathbf{c}$ : ${ }^{1} \mathrm{H}$ NMR $\left(\mathrm{CDCl}_{3}\right)$ 87.44-7.38 (m, 
4H), 5.69, (m, 1H), $5.58(\mathrm{~m}, 1 \mathrm{H}), 3.75(\mathrm{~s}, 2 \mathrm{H}), 3.17(\mathrm{~m}, 1 \mathrm{H}), 3.12$ (t. $1 \mathrm{H}, J=5.2 \mathrm{~Hz}), 2.42$ (dd, $1 \mathrm{H}, J=16.4,2.0 \mathrm{~Hz}), 2.07(\mathrm{~m}, 1 \mathrm{H}), 1.92(\mathrm{~m}, 1 \mathrm{H}), 1.81(\mathrm{~m}, 1 \mathrm{H}), 1.61-1.53(\mathrm{~m}, 2 \mathrm{H}) ;{ }^{13} \mathrm{C} \mathrm{NMR}$ $\left(\mathrm{CDCl}_{3}\right) \delta 145.0,131.3,129.8,129.5,126.3,125.7$ (q, $\left.J_{\mathrm{C}-\mathrm{F}}=3.7 \mathrm{~Hz}\right), 124.3,57.2,56.4,53.3,34.8$, 32.2, 30.7; GCMS (m/z) 267, 238, 159; Anal. Calcd. for $\mathrm{C}_{15} \mathrm{H}_{16} \mathrm{~F}_{3} \mathrm{~N}$ : C, 67.40; H, 6.03; N, 5.24; Found for C, 67.60; H, 6.05; N, 5.46

8-phenylethyl-8-aza-bicyclo[3.2.1]oct-2-ene (6d). General procedure as above with phenethylamine $(0.500 \mathrm{mmol}, 53.6 \mathrm{mg})$, triene $(2.00 \mathrm{mmol}, 207 \mu \mathrm{L})$ in toluene $(0.5 \mathrm{~mL})$ gave 77 mg $(72 \%)$ of $\mathbf{6 d}$ as yellow oil. $\mathbf{6 d}$ : ${ }^{1} \mathrm{H}$ NMR $\left(\mathrm{CDCl}_{3}\right)$ 87.35-7.22 (m, 4H), 5.82, (m, 1H), $5.63(\mathrm{~m}$, $1 \mathrm{H}), 3.43$ (m, 2H), 2.93-2.85 (m. 2H), 2.84-2.80 (m, 2H), 2.56 (dd, 1H, J = 10.4, $2.0 \mathrm{~Hz}), 2.21$ (m, 1H), $2.08(\mathrm{~m}, 1 \mathrm{H}), 1.92(\mathrm{~m}, 1 \mathrm{H}), 1.72-1.64(\mathrm{~m}, 2 \mathrm{H}) ;{ }^{13} \mathrm{C} \mathrm{NMR}\left(\mathrm{CDCl}_{3}\right) \delta 141.2,131.3,129.3$, 128.9, 126.6, 124.2, 57.6, 56.7, 51.9, 36.4, 34.6, 32.3, 30.4; GCMS ( $m / z)$ 213; Anal. Calcd. for $\mathrm{C}_{15} \mathrm{H}_{19} \mathrm{~N}$ : C, 84.46; H, 8.98; N, 6.57; Found for C, 84.19; H, 9.21; N, 6.59.

Crossover reaction of 8-(4-methylphenyl)-8-aza-bicyclo[3.2.1]oct-2-ene (3g) and 4-tert-butylaniline (2l). In a glovebox, $\operatorname{Pd}(\mathrm{TFA})_{2}(2 \mathrm{~mol} \%, 2.0 \mathrm{mg})$ and Xantphos (4 mol\%, 7.0 $\mathrm{mg}$ ), and benzoic acid (10 mol\%, $3.7 \mathrm{mg})$ were weighed directly into a screw capped vial. A stirrer bar, 8-(4-methylphenyl)-8-aza-bicyclo[3.2.1]oct-2-ene (3g) (0.300 mmol, $45.1 \mathrm{mg}$ ), 4-tert-butylamine $(\mathbf{2 l}, 0.300 \mathrm{mmol}, 44.8 \mathrm{mg})$, dodecane $(0.125 \mathrm{mmol}, 21.3 \mathrm{mg})$ as an internal standard, and toluene $(0.6 \mathrm{~mL})$ were added and the reaction mixture removed from the glovebox. The reaction mixture was stirred at $110{ }^{\circ} \mathrm{C}$. Aliquots $(5 \mu \mathrm{L})$ were taken at various times, these aliquots were diluted to $0.5 \mathrm{~mL}$ with dichloromethane and analyzed by gas chromatography. The GC yields were calculated from the ratio of peal areas of the reaction components and the internal standard along with response factors obtained by isolated compounds. 


\section{Experimental Details for crystal structure determination of 3a.}

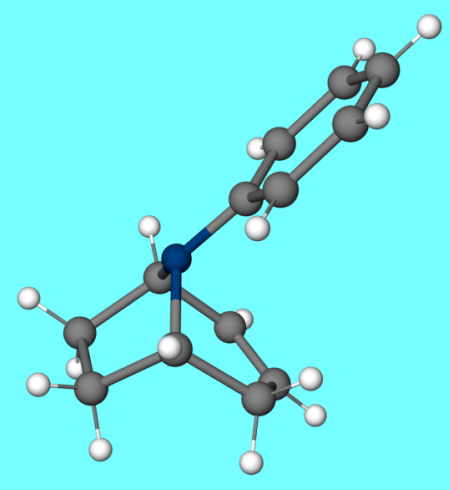

\section{Data Collection}

A colorless plate crystal of $\mathrm{C}_{13} \mathrm{H}_{15} \mathrm{~N}$ having approximate dimensions of $0.25 \times 0.25 \times 0.10 \mathrm{~mm}^{3}$ was mounted with epoxy cement on the tip of a fine glass fiber. All measurements were made on a Nonius KappaCCD diffractometer with graphite monochromated Mo-K $\alpha$ radiation.

Cell constants and an orientation matrix for data collection corresponded to a primitive monoclinic cell with dimensions:

$$
\begin{aligned}
& \mathrm{a}=9.1032(18) \AA \quad \alpha=90^{\circ} \\
& \mathrm{b}=14.528(3) \AA \beta=102.46(3)^{\mathrm{o}} \\
& \mathrm{c}=15.888(3) \AA \gamma=90^{\circ} \\
& \mathrm{V}=2051.8(7) \AA^{3}
\end{aligned}
$$

For $\mathrm{Z}=8$ and F.W. $=185.26$, the calculated density is $1.199 \mathrm{~g} / \mathrm{cm}^{3}$. Based on a statistical analysis of intensity distribution, and the successful solution and refinement of the structure, the space group was determined to be $P 2_{1} / n(\# 14)$.

The data were collected at a temperature of $173(2) \mathrm{K}$ to a maximum $2 \theta$ value of $56.52^{\circ}$. Five omega scans consisting of $29,37,35,15$, and 14 data frames, respectively, were collected with a frame width of $2.0^{\circ}$ and a detector-to-crystal distance, Dx, of $35.0 \mathrm{~mm}$. Each frame was exposed twice (for the purpose of de-zingering) for a total of $120 \mathrm{~s}$. The data frames were processed and scaled using the DENZO software package. ${ }^{1}$

\section{Data Reduction}

A total of 8480 reflections were collected of which 5030 were unique and observed $\left(R_{\text {int }}=0.0875\right)$. The linear absorption coefficient, $\mu$, for Mo-K $\alpha$ radiation is $0.70 \mathrm{~cm}^{-1}$, and no absorption correction was applied. The data were corrected for Lorentz and polarization effects.

\section{$\underline{\text { Structure Solution and Refinement }}$}

The structure was solved by direct methods and expanded using Fourier techniques ${ }^{2}$. The non-hydrogen atoms were refined anisotropically, and hydrogen atoms were treated as idealized contributions. The final cycle of 
full-matrix least-squares refinement ${ }^{3}$ on $\mathrm{F}$ was based on 5030 observed reflections (I > 2.00๘(I)) and 253 variable parameters and converged with unweighted and weighted agreement factors of:

$$
\begin{gathered}
\mathrm{R}=\Sigma\|\mathrm{Fol}-|\mathrm{Fc} \| / \Sigma| \mathrm{Fo} \mid=0.0624 \\
\mathrm{R}_{\mathrm{W}}=\left\{\Sigma\left[\mathrm{w}\left(\mathrm{F}_{\mathrm{o}}{ }^{2}-\mathrm{F}_{\mathrm{c}}\right)^{2}\right] / \Sigma\left[\mathrm{w}\left(\mathrm{F}_{\mathrm{o}}^{2}\right)^{2}\right]\right\}^{1 / 2}=0.1006
\end{gathered}
$$

The maximum and minimum peaks on the final difference Fourier map corresponded to 0.227 and -0.240 $\mathrm{e}^{-} / \AA^{3}$ respectively.

\section{REFERENCES}

(1) Z. Otwinowski and W. Minor, "Processing of X-Ray Diffraction Data Collected in Oscillation Mode," Methods in Enzymology, vol. 276: Macromolecular Crystallography, part A, 307-326, 1997, C.W. Carter, Jr. \& R.M. Sweet, Eds., Academic Press.

(2) Acta Cryst. A46 (1990) 467-473

(3) Least Squares function minimized: $\quad \Sigma w\left(\mathrm{~F}_{\mathrm{o}}^{2}-\mathrm{F}_{\mathrm{c}}\right)^{2}$

Table S1. Crystal data and structure refinement for $\mathbf{3 a}$

$\begin{array}{lll}\text { Empirical formula } & \mathrm{C}_{13} \mathrm{H}_{15} \mathrm{~N} & \\ \text { Formula weight } & 185.26 \\ \text { Temperature } & 173(2) \mathrm{K} \\ \text { Wavelength } & 0.71073 \AA \\ \text { Crystal system } & \text { Monoclinic } & \\ \text { Space group } & \mathrm{P} 2(1) / \mathrm{n} & \alpha=90^{\circ} . \\ \text { Unit cell dimensions } & \mathrm{a}=9.1032(18) \AA & \beta=102.46(3)^{\circ} . \\ & \mathrm{b}=14.528(3) \AA & \gamma=90^{\circ} . \\ & \mathrm{c}=15.888(3) \AA & \\ \text { Volume } & 2051.8(7) \AA^{3} & \\ \text { Z } & 8 & \\ \text { Density (calculated) } & 1.199 \mathrm{~g} / \mathrm{cm}^{3} & \\ \text { Absorption coefficient } & 0.70 \mathrm{~cm}^{-1} \\ \text { F(000) } & 800 \\ \text { Crystal size } & 0.25 \mathrm{x} 0.25 \times 0.10 \mathrm{~mm}^{3} \\ \text { Theta range for data collection } & 2.63 \text { to } 28.26^{\circ} . \\ \text { Index ranges } & -12<=\mathrm{h}<=12,-19<=\mathrm{k}<=19,-21<=1<=21 \\ \text { Reflections collected } & 8480 \\ \text { Independent reflections } & 5030[\mathrm{R}(\mathrm{int})=0.0875] \\ \text { Completeness to theta }=28.26^{\circ} & 99.2 \% \\ \end{array}$


Absorption correction

Max. and min. transmission

Refinement method

Data / restraints / parameters

Goodness-of-fit on $\mathrm{F}^{2}$

Final $R$ indices [I $>2 \operatorname{sigma}(\mathrm{I})]$

$\mathrm{R}$ indices (all data)

Largest diff. peak and hole
None

0.9931 and 0.9828

Full-matrix least-squares on $\mathrm{F}^{2}$

$5030 / 0 / 253$

1.004

$\mathrm{R} 1=0.0624, \mathrm{wR} 2=0.1006$

$\mathrm{R} 1=0.1910, \mathrm{wR} 2=0.1325$

0.227 and -0.240 e. $\AA^{-3}$ 
Table S2. Atomic coordinates $\left(\mathrm{x} 10^{4}\right)$ and equivalent isotropic displacement parameters $\left(\AA^{2} \times 10^{3}\right)$ for $3 \mathbf{a}$. $\mathrm{U}(\mathrm{eq})$ is defined as one third of the trace of the orthogonalized $\mathrm{U}^{\mathrm{ij}}$ tensor.

\begin{tabular}{|c|c|c|c|c|}
\hline & $\mathrm{x}$ & $\mathrm{y}$ & $\mathrm{z}$ & $\mathrm{U}(\mathrm{eq})$ \\
\hline $\mathrm{N}(1)$ & $2396(2)$ & 4912(1) & 1671(1) & $27(1)$ \\
\hline$C(1)$ & $3989(2)$ & $4657(2)$ & 2018(1) & $33(1)$ \\
\hline$C(2)$ & $4667(2)$ & $4740(2)$ & $1217(1)$ & $41(1)$ \\
\hline$C(3)$ & $3614(2)$ & $5396(2)$ & $609(1)$ & $39(1)$ \\
\hline$C(4)$ & $2544(2)$ & $5743(1)$ & 1161(1) & $30(1)$ \\
\hline$C(5)$ & $3276(2)$ & $6507(2)$ & 1741(1) & $42(1)$ \\
\hline$C(6)$ & $4306(3)$ & $6298(2)$ & $2465(2)$ & $47(1)$ \\
\hline$C(7)$ & $4688(2)$ & $5341(2)$ & 2715(1) & $45(1)$ \\
\hline$C(8)$ & $1380(2)$ & 4916(1) & $2225(1)$ & $26(1)$ \\
\hline $\mathrm{C}(9)$ & $1438(2)$ & 4221(1) & $2840(1)$ & $30(1)$ \\
\hline$C(10)$ & $406(2)$ & $4189(2)$ & $3359(1)$ & $33(1)$ \\
\hline $\mathrm{C}(11)$ & $-714(2)$ & $4840(2)$ & $3285(1)$ & $34(1)$ \\
\hline$C(12)$ & $-800(2)$ & $5522(2)$ & 2674(1) & $36(1)$ \\
\hline$C(13)$ & $230(2)$ & $5563(1)$ & $2147(1)$ & $31(1)$ \\
\hline $\mathrm{N}\left(1^{\prime}\right)$ & $-5398(2)$ & 7559(1) & $5313(1)$ & $27(1)$ \\
\hline$C\left(1^{\prime}\right)$ & $-6939(2)$ & $7895(2)$ & 4956(1) & $37(1)$ \\
\hline$C\left(2^{\prime}\right)$ & $-7673(2)$ & $7826(2)$ & $5740(1)$ & $48(1)$ \\
\hline$C\left(3^{\prime}\right)$ & $-6686(3)$ & $7142(2)$ & $6357(1)$ & $45(1)$ \\
\hline$C\left(4^{\prime}\right)$ & $-5676(2)$ & $6735(1)$ & $5797(1)$ & $35(1)$ \\
\hline$C\left(5^{\prime}\right)$ & $-6499(2)$ & $6012(2)$ & $5188(2)$ & $42(1)$ \\
\hline$C\left(6^{\prime}\right)$ & $-7433(3)$ & $6282(2)$ & $4458(2)$ & $49(1)$ \\
\hline$C\left(7^{\prime}\right)$ & $-7692(2)$ & $7258(2)$ & 4222(1) & $48(1)$ \\
\hline$C\left(8^{\prime}\right)$ & $-4356(2)$ & $7520(1)$ & $4780(1)$ & $25(1)$ \\
\hline $\mathrm{C}\left(9^{\prime}\right)$ & $-4329(2)$ & $8221(1)$ & 4179(1) & $31(1)$ \\
\hline$C\left(10^{\prime}\right)$ & $-3245(2)$ & $8245(2)$ & $3692(1)$ & $36(1)$ \\
\hline$C\left(11^{\prime}\right)$ & $-2150(2)$ & $7573(2)$ & $3789(1)$ & $38(1)$ \\
\hline$C\left(12^{\prime}\right)$ & $-2161(2)$ & $6876(2)$ & $4376(1)$ & $37(1)$ \\
\hline$C\left(13^{\prime}\right)$ & $-3254(2)$ & 6841(2) & $4865(1)$ & $33(1)$ \\
\hline
\end{tabular}


Table S3. Bond lengths $[\AA]$ and angles $\left[{ }^{\circ}\right]$ for $\mathbf{3 a}$

\begin{tabular}{|c|c|}
\hline $\mathrm{N}(1)-\mathrm{C}(8)$ & $1.408(2)$ \\
\hline $\mathrm{N}(1)-\mathrm{C}(4)$ & $1.476(2)$ \\
\hline $\mathrm{N}(1)-\mathrm{C}(1)$ & $1.483(2)$ \\
\hline $\mathrm{C}(1)-\mathrm{C}(7)$ & $1.521(3)$ \\
\hline$C(1)-C(2)$ & $1.535(3)$ \\
\hline$C(2)-C(3)$ & $1.537(3)$ \\
\hline $\mathrm{C}(3)-\mathrm{C}(4)$ & $1.531(3)$ \\
\hline$C(4)-C(5)$ & $1.503(3)$ \\
\hline$C(5)-C(6)$ & $1.353(3)$ \\
\hline$C(6)-C(7)$ & $1.466(3)$ \\
\hline$C(8)-C(13)$ & $1.392(3)$ \\
\hline $\mathrm{C}(8)-\mathrm{C}(9)$ & $1.398(3)$ \\
\hline$C(9)-C(10)$ & $1.378(3)$ \\
\hline $\mathrm{C}(10)-\mathrm{C}(11)$ & $1.377(3)$ \\
\hline $\mathrm{C}(11)-\mathrm{C}(12)$ & $1.378(3)$ \\
\hline$C(12)-C(13)$ & $1.387(3)$ \\
\hline $\mathrm{N}\left(1^{\prime}\right)-\mathrm{C}\left(8^{\prime}\right)$ & $1.403(2)$ \\
\hline $\mathrm{N}\left(1^{\prime}\right)-\mathrm{C}\left(4^{\prime}\right)$ & $1.474(2)$ \\
\hline $\mathrm{N}\left(1^{\prime}\right)-\mathrm{C}\left(1^{\prime}\right)$ & $1.479(2)$ \\
\hline$C\left(1^{\prime}\right)-C\left(7^{\prime}\right)$ & $1.531(3)$ \\
\hline $\mathrm{C}\left(1^{\prime}\right)-\mathrm{C}\left(2^{\prime}\right)$ & $1.537(3)$ \\
\hline $\mathrm{C}\left(2^{\prime}\right)-\mathrm{C}\left(3^{\prime}\right)$ & $1.542(3)$ \\
\hline$C\left(3^{\prime}\right)-C\left(4^{\prime}\right)$ & $1.530(3)$ \\
\hline$C\left(4^{\prime}\right)-C\left(5^{\prime}\right)$ & $1.512(3)$ \\
\hline$C\left(5^{\prime}\right)-C\left(6^{\prime}\right)$ & $1.340(3)$ \\
\hline $\mathrm{C}\left(6^{\prime}\right)-\mathrm{C}\left(7^{\prime}\right)$ & $1.474(3)$ \\
\hline$C\left(8^{\prime}\right)-C\left(13^{\prime}\right)$ & $1.393(3)$ \\
\hline $\mathrm{C}\left(8^{\prime}\right)-\mathrm{C}\left(9^{\prime}\right)$ & $1.399(3)$ \\
\hline $\mathrm{C}\left(9^{\prime}\right)-\mathrm{C}\left(10^{\prime}\right)$ & $1.380(3)$ \\
\hline$C\left(10^{\prime}\right)-C\left(11^{\prime}\right)$ & $1.380(3)$ \\
\hline $\mathrm{C}\left(11^{\prime}\right)-\mathrm{C}\left(12^{\prime}\right)$ & $1.377(3)$ \\
\hline $\mathrm{C}\left(12^{\prime}\right)-\mathrm{C}\left(13^{\prime}\right)$ & $1.390(3)$ \\
\hline $\mathrm{C}(8)-\mathrm{N}(1)-\mathrm{C}(4)$ & $119.35(16)$ \\
\hline $\mathrm{C}(8)-\mathrm{N}(1)-\mathrm{C}(1)$ & $119.37(16)$ \\
\hline $\mathrm{C}(4)-\mathrm{N}(1)-\mathrm{C}(1)$ & $102.20(15)$ \\
\hline $\mathrm{N}(1)-\mathrm{C}(1)-\mathrm{C}(7)$ & $109.19(17)$ \\
\hline $\mathrm{N}(1)-\mathrm{C}(1)-\mathrm{C}(2)$ & $102.00(16)$ \\
\hline$C(7)-C(1)-C(2)$ & $111.86(18)$ \\
\hline
\end{tabular}




\begin{tabular}{|c|c|}
\hline $\mathrm{C}(1)-\mathrm{C}(2)-\mathrm{C}(3)$ & $105.18(17)$ \\
\hline$C(4)-C(3)-C(2)$ & $103.41(16)$ \\
\hline $\mathrm{N}(1)-\mathrm{C}(4)-\mathrm{C}(5)$ & $110.40(16)$ \\
\hline $\mathrm{N}(1)-\mathrm{C}(4)-\mathrm{C}(3)$ & $100.96(16)$ \\
\hline$C(5)-C(4)-C(3)$ & $109.94(18)$ \\
\hline$C(6)-C(5)-C(4)$ & $119.3(2)$ \\
\hline$C(5)-C(6)-C(7)$ & $121.5(2)$ \\
\hline $\mathrm{C}(6)-\mathrm{C}(7)-\mathrm{C}(1)$ & $112.67(19)$ \\
\hline$C(13)-C(8)-C(9)$ & $117.69(19)$ \\
\hline $\mathrm{C}(13)-\mathrm{C}(8)-\mathrm{N}(1)$ & $121.96(18)$ \\
\hline $\mathrm{C}(9)-\mathrm{C}(8)-\mathrm{N}(1)$ & $120.21(19)$ \\
\hline $\mathrm{C}(10)-\mathrm{C}(9)-\mathrm{C}(8)$ & $121.0(2)$ \\
\hline $\mathrm{C}(11)-\mathrm{C}(10)-\mathrm{C}(9)$ & $120.9(2)$ \\
\hline $\mathrm{C}(10)-\mathrm{C}(11)-\mathrm{C}(12)$ & $118.8(2)$ \\
\hline $\mathrm{C}(11)-\mathrm{C}(12)-\mathrm{C}(13)$ & $120.9(2)$ \\
\hline $\mathrm{C}(12)-\mathrm{C}(13)-\mathrm{C}(8)$ & $120.7(2)$ \\
\hline $\mathrm{C}\left(8^{\prime}\right)-\mathrm{N}\left(1^{\prime}\right)-\mathrm{C}\left(4^{\prime}\right)$ & $119.85(17)$ \\
\hline $\mathrm{C}\left(8^{\prime}\right)-\mathrm{N}\left(1^{\prime}\right)-\mathrm{C}\left(1^{\prime}\right)$ & $119.41(16)$ \\
\hline $\mathrm{C}\left(4^{\prime}\right)-\mathrm{N}\left(1^{\prime}\right)-\mathrm{C}\left(1^{\prime}\right)$ & $102.28(16)$ \\
\hline $\mathrm{N}\left(1^{\prime}\right)-\mathrm{C}\left(1^{\prime}\right)-\mathrm{C}\left(7^{\prime}\right)$ & $108.87(17)$ \\
\hline $\mathrm{N}\left(1^{\prime}\right)-\mathrm{C}\left(1^{\prime}\right)-\mathrm{C}\left(2^{\prime}\right)$ & $102.13(16)$ \\
\hline$C\left(7^{\prime}\right)-C\left(1^{\prime}\right)-C\left(2^{\prime}\right)$ & $112.15(18)$ \\
\hline$C\left(1^{\prime}\right)-C\left(2^{\prime}\right)-C\left(3^{\prime}\right)$ & $105.20(17)$ \\
\hline $\mathrm{C}\left(4^{\prime}\right)-\mathrm{C}\left(3^{\prime}\right)-\mathrm{C}\left(2^{\prime}\right)$ & $102.70(16)$ \\
\hline $\mathrm{N}\left(1^{\prime}\right)-\mathrm{C}\left(4^{\prime}\right)-\mathrm{C}\left(5^{\prime}\right)$ & $110.33(16)$ \\
\hline $\mathrm{N}\left(1^{\prime}\right)-\mathrm{C}\left(4^{\prime}\right)-\mathrm{C}\left(3^{\prime}\right)$ & $100.50(17)$ \\
\hline$C\left(5^{\prime}\right)-C\left(4^{\prime}\right)-C\left(3^{\prime}\right)$ & $111.30(18)$ \\
\hline$C\left(6^{\prime}\right)-C\left(5^{\prime}\right)-C\left(4^{\prime}\right)$ & $119.0(2)$ \\
\hline $\mathrm{C}\left(5^{\prime}\right)-\mathrm{C}\left(6^{\prime}\right)-\mathrm{C}\left(7^{\prime}\right)$ & $122.6(2)$ \\
\hline $\mathrm{C}\left(6^{\prime}\right)-\mathrm{C}\left(7^{\prime}\right)-\mathrm{C}\left(1^{\prime}\right)$ & $111.54(18)$ \\
\hline $\mathrm{C}\left(13^{\prime}\right)-\mathrm{C}\left(8^{\prime}\right)-\mathrm{C}\left(9^{\prime}\right)$ & $117.63(19)$ \\
\hline $\mathrm{C}\left(13^{\prime}\right)-\mathrm{C}\left(8^{\prime}\right)-\mathrm{N}\left(1^{\prime}\right)$ & $122.40(18)$ \\
\hline $\mathrm{C}\left(9^{\prime}\right)-\mathrm{C}\left(8^{\prime}\right)-\mathrm{N}\left(1^{\prime}\right)$ & $119.79(19)$ \\
\hline $\mathrm{C}\left(10^{\prime}\right)-\mathrm{C}\left(9^{\prime}\right)-\mathrm{C}\left(8^{\prime}\right)$ & $121.4(2)$ \\
\hline$C\left(9^{\prime}\right)-C\left(10^{\prime}\right)-C\left(11^{\prime}\right)$ & $120.4(2)$ \\
\hline $\mathrm{C}\left(12^{\prime}\right)-\mathrm{C}\left(11^{\prime}\right)-\mathrm{C}\left(10^{\prime}\right)$ & 119.1(2) \\
\hline $\mathrm{C}\left(11^{\prime}\right)-\mathrm{C}\left(12^{\prime}\right)-\mathrm{C}\left(13^{\prime}\right)$ & $121.1(2)$ \\
\hline $\mathrm{C}\left(12^{\prime}\right)-\mathrm{C}\left(13^{\prime}\right)-\mathrm{C}\left(8^{\prime}\right)$ & $120.5(2)$ \\
\hline
\end{tabular}

Symmetry transformations used to generate equivalent atoms 
Table S4. Anisotropic displacement parameters $\left(\AA^{2} \times 10^{3}\right)$ for 3a. The anisotropic displacement factor exponent takes the form: $-2 \pi^{2}\left[h^{2} a^{* 2} U^{11}+\ldots+2 h k a^{*} b^{*} U^{12}\right]$

\begin{tabular}{|c|c|c|c|c|c|c|}
\hline & $\mathrm{U}^{11}$ & $\mathrm{U}^{22}$ & $\mathrm{U}^{33}$ & $\mathrm{U}^{23}$ & $\mathrm{U}^{13}$ & $\mathrm{U}^{12}$ \\
\hline $\mathrm{N}(1)$ & $28(1)$ & $25(1)$ & $27(1)$ & $4(1)$ & $7(1)$ & $1(1)$ \\
\hline$C(1)$ & $28(1)$ & $35(1)$ & $38(1)$ & $5(1)$ & 11(1) & $8(1)$ \\
\hline$C(2)$ & $40(1)$ & $40(1)$ & $47(1)$ & $4(1)$ & $20(1)$ & $2(1)$ \\
\hline C(3) & $44(2)$ & $39(1)$ & $36(1)$ & $3(1)$ & $15(1)$ & $0(1)$ \\
\hline$C(4)$ & $33(1)$ & $28(1)$ & $30(1)$ & $6(1)$ & $9(1)$ & $2(1)$ \\
\hline$C(5)$ & $50(2)$ & $28(1)$ & $53(2)$ & $-2(1)$ & 21(1) & $-4(1)$ \\
\hline$C(6)$ & $45(2)$ & $52(2)$ & $48(2)$ & $-15(1)$ & $19(1)$ & $-20(1)$ \\
\hline$C(7)$ & $29(1)$ & $65(2)$ & $39(1)$ & $4(1)$ & $6(1)$ & $-5(1)$ \\
\hline$C(8)$ & $28(1)$ & $25(1)$ & $25(1)$ & $-2(1)$ & $7(1)$ & $-4(1)$ \\
\hline$C(9)$ & $26(1)$ & $30(1)$ & $32(1)$ & $3(1)$ & $5(1)$ & $2(1)$ \\
\hline$C(10)$ & $33(1)$ & $37(1)$ & $30(1)$ & $5(1)$ & $6(1)$ & $-5(1)$ \\
\hline $\mathrm{C}(11)$ & $30(1)$ & $41(2)$ & $34(1)$ & $-4(1)$ & $13(1)$ & $-6(1)$ \\
\hline$C(12)$ & $32(1)$ & $34(1)$ & $44(1)$ & $-2(1)$ & $12(1)$ & $3(1)$ \\
\hline $\mathrm{C}(13)$ & $32(1)$ & $29(1)$ & $34(1)$ & $4(1)$ & $10(1)$ & $5(1)$ \\
\hline $\mathrm{N}\left(1^{\prime}\right)$ & $26(1)$ & $27(1)$ & $28(1)$ & $1(1)$ & $5(1)$ & $0(1)$ \\
\hline$C\left(1^{\prime}\right)$ & $28(1)$ & $37(1)$ & $47(1)$ & $1(1)$ & $10(1)$ & $6(1)$ \\
\hline$C\left(2^{\prime}\right)$ & $39(2)$ & $54(2)$ & $57(2)$ & $-14(1)$ & $23(1)$ & $-5(1)$ \\
\hline$C\left(3^{\prime}\right)$ & $55(2)$ & $45(2)$ & $44(1)$ & $-10(1)$ & $26(1)$ & $-17(1)$ \\
\hline$C\left(4^{\prime}\right)$ & $40(1)$ & $33(1)$ & $32(1)$ & 1(1) & $10(1)$ & $-5(1)$ \\
\hline$C\left(5^{\prime}\right)$ & $46(2)$ & $32(1)$ & $53(2)$ & $-6(1)$ & $22(1)$ & $-9(1)$ \\
\hline$C\left(6^{\prime}\right)$ & $41(2)$ & $57(2)$ & $52(2)$ & $-22(1)$ & $15(1)$ & $-18(1)$ \\
\hline$C\left(7^{\prime}\right)$ & $26(1)$ & $71(2)$ & $43(1)$ & 2(1) & $1(1)$ & $1(1)$ \\
\hline$C\left(8^{\prime}\right)$ & $25(1)$ & $28(1)$ & $23(1)$ & $-4(1)$ & $4(1)$ & $-4(1)$ \\
\hline $\mathrm{C}\left(9^{\prime}\right)$ & $31(1)$ & $32(1)$ & $30(1)$ & $2(1)$ & $4(1)$ & $-1(1)$ \\
\hline$C\left(10^{\prime}\right)$ & $37(1)$ & $41(1)$ & $29(1)$ & $3(1)$ & $7(1)$ & $-10(1)$ \\
\hline$C\left(11^{\prime}\right)$ & $31(1)$ & $51(2)$ & $33(1)$ & $-4(1)$ & $9(1)$ & $-4(1)$ \\
\hline$C\left(12^{\prime}\right)$ & $30(1)$ & $43(2)$ & $38(1)$ & $-5(1)$ & $6(1)$ & $7(1)$ \\
\hline$C\left(13^{\prime}\right)$ & $36(1)$ & $32(1)$ & $29(1)$ & $2(1)$ & $6(1)$ & $6(1)$ \\
\hline
\end{tabular}


Table S5. Hydrogen coordinates ( $\left.\times 10^{4}\right)$ and isotropic displacement parameters $\left(\AA^{2} \times 10^{3}\right)$ for $3 \mathbf{a}$.

\begin{tabular}{|c|c|c|c|c|}
\hline & $\mathrm{x}$ & $\mathrm{y}$ & z & $\mathrm{U}(\mathrm{eq})$ \\
\hline $\mathrm{H}(1 \mathrm{~A})$ & 4073 & 4013 & 2246 & 40 \\
\hline $\mathrm{H}(2 \mathrm{~A})$ & 4705 & 4131 & 942 & 49 \\
\hline $\mathrm{H}(2 \mathrm{~B})$ & 5699 & 4994 & 1371 & 49 \\
\hline $\mathrm{H}(3 \mathrm{~A})$ & 4183 & 5911 & 424 & 46 \\
\hline $\mathrm{H}(3 \mathrm{~B})$ & 3058 & 5067 & 92 & 46 \\
\hline $\mathrm{H}(4 \mathrm{~A})$ & 1553 & 5937 & 799 & 36 \\
\hline $\mathrm{H}(5 \mathrm{~A})$ & 3019 & 7130 & 1599 & 50 \\
\hline $\mathrm{H}(6 \mathrm{~A})$ & 4793 & 6782 & 2821 & 56 \\
\hline $\mathrm{H}(7 \mathrm{~A})$ & 4338 & 5200 & 3249 & 53 \\
\hline $\mathrm{H}(7 \mathrm{~B})$ & 5795 & 5269 & 2841 & 53 \\
\hline $\mathrm{H}(9 \mathrm{~A})$ & 2201 & 3764 & 2901 & 35 \\
\hline $\mathrm{H}(10 \mathrm{~A})$ & 468 & 3711 & 3773 & 40 \\
\hline $\mathrm{H}(11 \mathrm{~A})$ & -1415 & 4819 & 3649 & 41 \\
\hline $\mathrm{H}(12 \mathrm{~A})$ & -1576 & 5970 & 2612 & 43 \\
\hline $\mathrm{H}(13 \mathrm{~A})$ & 151 & 6037 & 1728 & 38 \\
\hline $\mathrm{H}\left(1^{\prime} \mathrm{A}\right)$ & -6926 & 8546 & 4753 & 44 \\
\hline $\mathrm{H}\left(2^{\prime} \mathrm{A}\right)$ & -8718 & 7597 & 5565 & 58 \\
\hline $\mathrm{H}\left(2^{\prime} \mathrm{B}\right)$ & -7687 & 8435 & 6018 & 58 \\
\hline $\mathrm{H}\left(3^{\prime} \mathrm{A}\right)$ & -6085 & 7460 & 6867 & 55 \\
\hline $\mathrm{H}\left(3^{\prime} \mathrm{B}\right)$ & -7307 & 6659 & 6551 & 55 \\
\hline $\mathrm{H}\left(4^{\prime} \mathrm{A}\right)$ & -4720 & 6486 & 6157 & 42 \\
\hline $\mathrm{H}\left(5^{\prime} \mathrm{A}\right)$ & -6357 & 5377 & 5321 & 51 \\
\hline $\mathrm{H}\left(6^{\prime} \mathrm{A}\right)$ & -7949 & 5825 & 4078 & 59 \\
\hline $\mathrm{H}\left(7^{\prime} \mathrm{A}\right)$ & -8787 & 7382 & 4076 & 57 \\
\hline $\mathrm{H}\left(7^{\prime} \mathrm{B}\right)$ & -7286 & 7390 & 3704 & 57 \\
\hline $\mathrm{H}\left(9^{\prime} \mathrm{A}\right)$ & -5072 & 8691 & 4106 & 38 \\
\hline $\mathrm{H}(10 \mathrm{~B})$ & -3253 & 8727 & 3287 & 43 \\
\hline $\mathrm{H}(11 \mathrm{~B})$ & -1400 & 7591 & 3457 & 45 \\
\hline $\mathrm{H}(12 \mathrm{~B})$ & -1409 & 6412 & 4447 & 44 \\
\hline $\mathrm{H}(13 \mathrm{C})$ & -3250 & 6350 & 5260 & \\
\hline
\end{tabular}




\section{Experimental Details for crystal structure determination of $4 c$.}

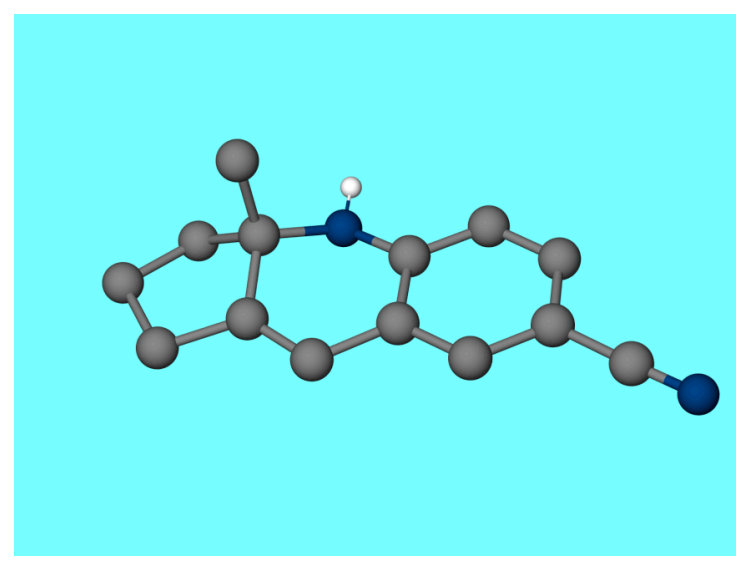

\section{Data Collection}

A pale yellow block crystal of $\mathrm{C}_{14} \mathrm{H}_{14} \mathrm{~N}_{2}$ having approximate dimensions of $0.20 \times 0.10 \times 0.08 \mathrm{~mm}^{3}$ was mounted with epoxy cement on the tip of a fine glass fiber. All measurements were made on a Nonius KappaCCD diffractometer with graphite monochromated Mo-Ka radiation.

Cell constants and an orientation matrix for data collection corresponded to a primitive orthorhombic cell with dimensions:

$$
\begin{aligned}
& \mathrm{a}=17.678(4) \AA \alpha=90^{\circ} \\
& \mathrm{b}=5.8367(12) \AA \quad \beta=90^{\circ} \\
& \mathrm{c}=10.929(2) \AA \gamma=90^{\circ} \\
& \mathrm{V}=1127.7(4) \AA^{3}
\end{aligned}
$$

For $\mathrm{Z}=4$ and F.W. $=210.27$, the calculated density is $1.239 \mathrm{~g} / \mathrm{cm}^{3}$. Based on a statistical analysis of intensity distribution, and the successful solution and refinement of the structure, the space group was determined to be $P c a 2_{1}(\# 29)$.

The data were collected at a temperature of $173(2) \mathrm{K}$ to a maximum $2 \theta$ value of $56.58^{\circ}$. Four omega scans consisting of $35,32,31$, and 5 data frames, respectively, were collected with a frame width of $1.7^{\circ}$ and a detector-to-crystal distance, Dx, of $35.0 \mathrm{~mm}$. Each frame was exposed twice (for the purpose of de-zingering) for a total of $136 \mathrm{~s}$. The data frames were processed and scaled using the DENZO software package. ${ }^{1}$

\section{Data Reduction}

A total of 2507 reflections were collected of which 2507 were unique and observed $\left(R_{\text {int }}=0.00\right.$, Friedel pairs not merged). The linear absorption coefficient, $\mu$, for Mo-K $\alpha$ radiation is $0.74 \mathrm{~cm}^{-1}$, and no absorption correction 
was applied. The data were corrected for Lorentz and polarization effects.

\section{$\underline{\text { Structure Solution and Refinement }}$}

The structure was solved by direct methods and expanded using Fourier techniques ${ }^{2}$. The non-hydrogen atoms were refined anisotropically, and hydrogen atoms, with exceptions noted, were treated as idealized contributions. The final cycle of full-matrix least-squares refinement ${ }^{3}$ on $\mathrm{F}$ was based on 2507 observed reflections $(\mathrm{I}>2.00 \sigma(\mathrm{I}))$ and 149 variable parameters and converged with unweighted and weighted agreement factors of:

$$
\begin{gathered}
\mathrm{R}=\Sigma\|\mathrm{Fol}-|\mathrm{Fc} \| / \Sigma| \mathrm{Fo} \mid=0.0517 \\
\mathrm{R}_{\mathrm{W}}=\left\{\Sigma\left[\mathrm{w}\left(\mathrm{F}_{\mathrm{o}}^{2}-\mathrm{F}_{\mathrm{c}}\right)^{2}\right] / \Sigma\left[\mathrm{w}\left(\mathrm{F}_{\mathrm{o}}^{2}\right)^{2}\right]\right\}^{1 / 2}=0.1178
\end{gathered}
$$

The maximum and minimum peaks on the final difference Fourier map corresponded to 0.184 and -0.185 $\mathrm{e}^{-} / \AA^{3}$ respectively.

\section{REFERENCES}

(1) Z. Otwinowski and W. Minor, "Processing of X-Ray Diffraction Data Collected in Oscillation Mode," Methods in Enzymology, vol. 276: Macromolecular Crystallography, part A, 307-326, 1997, C.W. Carter, Jr. \& R.M. Sweet, Eds., Academic Press.

(2) Acta Cryst. A46 (1990) 467-473

(3) Least Squares function minimized: $\quad \Sigma w\left(\mathrm{~F}_{\mathrm{o}}{ }^{2}-\mathrm{F}_{\mathrm{c}}{ }^{2}\right)^{2}$ 
Table S6. Crystal data and structure refinement for $\mathbf{4 c .}$

Empirical formula

Formula weight

Temperature

Wavelength

Crystal system

Space group

Unit cell dimensions

Volume

Z

Density (calculated)

Absorption coefficient

$\mathrm{F}(000)$

Crystal size

Theta range for data collection

Index ranges

Reflections collected

Independent reflections

Completeness to theta $=28.29^{\circ}$

Absorption correction

Max. and min. transmission

Refinement method

Data / restraints / parameters

Goodness-of-fit on $\mathrm{F}^{2}$

Final $\mathrm{R}$ indices $[\mathrm{I}>2 \operatorname{sigma}(\mathrm{I})]$

$\mathrm{R}$ indices (all data)

Absolute structure parameter

Largest diff. peak and hole
$\mathrm{C}_{14} \mathrm{H}_{14} \mathrm{~N}_{2}$

210.27

173(2) K

$0.71073 \AA$

Orthorhombic

Pca2(1)

$\mathrm{a}=17.678(4) \AA$

$\alpha=90^{\circ}$.

$\mathrm{b}=5.8367(12) \AA$

$\beta=90^{\circ}$.

$\mathrm{c}=10.929(2) \AA$

$\gamma=90^{\circ}$.

1127.7(4) $\AA^{3}$

4

$1.239 \mathrm{~g} / \mathrm{cm}^{3}$

$0.74 \mathrm{~cm}^{-1}$

448

$0.20 \times 0.10 \times 0.08 \mathrm{~mm}^{3}$

2.30 to $28.29^{\circ}$.

$-23<=\mathrm{h}<=23,-7<=\mathrm{k}<=7,-14<=1<=14$

2507

$2507[\mathrm{R}(\mathrm{int})=0.0000]$

$99.4 \%$

None

0.9941 and 0.9853

Full-matrix least-squares on $\mathrm{F}^{2}$

$2507 / 1 / 149$

1.004

$\mathrm{R} 1=0.0517, \mathrm{wR} 2=0.1178$

$\mathrm{R} 1=0.0775, \mathrm{wR} 2=0.1297$

$-2(4)$

0.184 and -0.185 e. $\AA^{-3}$ 
Table S7. Atomic coordinates $\left(\mathrm{x} 10^{4}\right)$ and equivalent isotropic displacement parameters $\left(\AA^{2} \times 10^{3}\right)$ for 4 c. $\mathrm{U}(\mathrm{eq})$ is defined as one third of the trace of the orthogonalized $\mathrm{U}^{\mathrm{ij}}$ tensor.

\begin{tabular}{lrrrr}
\hline & $\mathrm{x}$ & $\mathrm{y}$ & $\mathrm{z}$ & $\mathrm{U}(\mathrm{eq})$ \\
\hline $\mathrm{N}(1)$ & & & & \\
$\mathrm{N}(2)$ & $4152(1)$ & $5567(3)$ & $6430(2)$ & $28(1)$ \\
$\mathrm{C}(1)$ & $1869(1)$ & $-1344(4)$ & $9730(2)$ & $51(1)$ \\
$\mathrm{C}(2)$ & $3673(1)$ & $4106(4)$ & $7044(2)$ & $25(1)$ \\
$\mathrm{C}(4)$ & $2929(1)$ & $4718(4)$ & $7303(2)$ & $30(1)$ \\
$\mathrm{C}(3)$ & $2742(1)$ & $1230(4)$ & $8425(2)$ & $30(1)$ \\
$\mathrm{C}(5)$ & $2467(1)$ & $3317(3)$ & $7990(2)$ & $32(1)$ \\
$\mathrm{C}(6)$ & $3487(1)$ & $586(4)$ & $8172(2)$ & $27(1)$ \\
$\mathrm{C}(7)$ & $3962(1)$ & $1999(3)$ & $7494(2)$ & $24(1)$ \\
$\mathrm{C}(8)$ & $4761(1)$ & $1497(4)$ & $7295(2)$ & $27(1)$ \\
$\mathrm{C}(9)$ & $5166(1)$ & $2752(3)$ & $6523(2)$ & $24(1)$ \\
$\mathrm{C}(10)$ & $6011(1)$ & $2728(4)$ & $6283(2)$ & $32(1)$ \\
$\mathrm{C}(11)$ & $6158(1)$ & $4856(4)$ & $5485(2)$ & $32(1)$ \\
$\mathrm{C}(12)$ & $5457(1)$ & $6374(3)$ & $5674(2)$ & $28(1)$ \\
$\mathrm{C}(13)$ & $4810(1)$ & $4644(3)$ & $5776(2)$ & $25(1)$ \\
$\mathrm{C}(14)$ & $2266(1)$ & $-209(4)$ & $9151(2)$ & $37(1)$ \\
& $4573(1)$ & $3761(4)$ & $4510(2)$ & $35(1)$ \\
\hline
\end{tabular}


Table S8. Bond lengths $[\AA]$ and angles $\left[{ }^{\circ}\right]$ for $\mathbf{4 c .}$

\begin{tabular}{|c|c|c|c|}
\hline $\mathrm{N}(1)-\mathrm{H}(1)$ & $0.85(3)$ & $\mathrm{C}(12)-\mathrm{C}(8)-\mathrm{C}(9)$ & $108.87(17)$ \\
\hline $\mathrm{N}(1)-\mathrm{C}(1)$ & $1.375(3)$ & $\mathrm{C}(8)-\mathrm{C}(9)-\mathrm{C}(10)$ & $104.82(16)$ \\
\hline $\mathrm{N}(1)-\mathrm{C}(12)$ & $1.468(3)$ & $C(11)-C(10)-C(9)$ & $104.70(16)$ \\
\hline $\mathrm{N}(2)-\mathrm{C}(13)$ & $1.153(3)$ & $\mathrm{C}(12)-\mathrm{C}(11)-\mathrm{C}(10)$ & $103.38(16)$ \\
\hline$C(1)-C(2)$ & $1.392(3)$ & $\mathrm{N}(1)-\mathrm{C}(12)-\mathrm{C}(8)$ & $109.57(16)$ \\
\hline $\mathrm{C}(1)-\mathrm{C}(6)$ & $1.420(3)$ & $\mathrm{N}(1)-\mathrm{C}(12)-\mathrm{C}(11)$ & $112.69(17)$ \\
\hline $\mathrm{C}(2)-\mathrm{C}(3)$ & $1.378(3)$ & $\mathrm{C}(8)-\mathrm{C}(12)-\mathrm{C}(11)$ & $102.17(15)$ \\
\hline $\mathrm{C}(4)-\mathrm{C}(3)$ & $1.395(3)$ & $\mathrm{N}(1)-\mathrm{C}(12)-\mathrm{C}(14)$ & $110.19(17)$ \\
\hline $\mathrm{C}(4)-\mathrm{C}(5)$ & $1.397(3)$ & $\mathrm{C}(8)-\mathrm{C}(12)-\mathrm{C}(14)$ & $110.84(17)$ \\
\hline$C(4)-C(13)$ & $1.430(3)$ & $\mathrm{C}(11)-\mathrm{C}(12)-\mathrm{C}(14)$ & $111.14(18)$ \\
\hline $\mathrm{C}(5)-\mathrm{C}(6)$ & $1.391(3)$ & $\mathrm{N}(2)-\mathrm{C}(13)-\mathrm{C}(4)$ & $178.7(3)$ \\
\hline$C(6)-C(7)$ & $1.459(3)$ & & \\
\hline $\mathrm{C}(7)-\mathrm{C}(8)$ & $1.327(3)$ & & \\
\hline$C(8)-C(12)$ & $1.510(3)$ & \multirow{2}{*}{\multicolumn{2}{|c|}{$\begin{array}{l}\text { Symmetry transformations used to generate equivalent } \\
\text { atoms }\end{array}$}} \\
\hline $\mathrm{C}(8)-\mathrm{C}(9)$ & $1.517(3)$ & & \\
\hline $\mathrm{C}(9)-\mathrm{C}(10)$ & $1.540(3)$ & & \\
\hline $\mathrm{C}(10)-\mathrm{C}(11)$ & $1.537(3)$ & & \\
\hline$C(11)-C(12)$ & $1.530(3)$ & & \\
\hline$C(12)-C(14)$ & $1.534(3)$ & & \\
\hline $\mathrm{H}(1)-\mathrm{N}(1)-\mathrm{C}(1)$ & $116.7(16)$ & & \\
\hline $\mathrm{H}(1)-\mathrm{N}(1)-\mathrm{C}(12)$ & $114.1(16)$ & & \\
\hline $\mathrm{C}(1)-\mathrm{N}(1)-\mathrm{C}(12)$ & $119.84(17)$ & & \\
\hline $\mathrm{N}(1)-\mathrm{C}(1)-\mathrm{C}(2)$ & $121.46(18)$ & & \\
\hline $\mathrm{N}(1)-\mathrm{C}(1)-\mathrm{C}(6)$ & $119.00(17)$ & & \\
\hline$C(2)-C(1)-C(6)$ & $119.40(18)$ & & \\
\hline$C(3)-C(2)-C(1)$ & $121.26(19)$ & & \\
\hline$C(3)-C(4)-C(5)$ & $119.7(2)$ & & \\
\hline$C(3)-C(4)-C(13)$ & $119.79(19)$ & & \\
\hline$C(5)-C(4)-C(13)$ & $120.5(2)$ & & \\
\hline$C(2)-C(3)-C(4)$ & $119.8(2)$ & & \\
\hline$C(6)-C(5)-C(4)$ & $121.01(19)$ & & \\
\hline $\mathrm{C}(5)-\mathrm{C}(6)-\mathrm{C}(1)$ & $118.77(18)$ & & \\
\hline$C(5)-C(6)-C(7)$ & $123.02(18)$ & & \\
\hline $\mathrm{C}(1)-\mathrm{C}(6)-\mathrm{C}(7)$ & $118.07(18)$ & & \\
\hline $\mathrm{C}(8)-\mathrm{C}(7)-\mathrm{C}(6)$ & $120.40(18)$ & & \\
\hline $\mathrm{C}(7)-\mathrm{C}(8)-\mathrm{C}(12)$ & $121.52(17)$ & & \\
\hline $\mathrm{C}(7)-\mathrm{C}(8)-\mathrm{C}(9)$ & $129.48(18)$ & & \\
\hline
\end{tabular}


Table S9. Anisotropic displacement parameters $\left(\AA^{2} \times 10^{3}\right)$ for $\mathbf{4 c} . \quad$ The anisotropic displacement factor exponent takes the form: $-2 \pi^{2}\left[h^{2} a^{* 2} U^{11}+\ldots+2 h k a^{*} b^{*} U^{12}\right]$

\begin{tabular}{|c|c|c|c|c|c|c|}
\hline & $\mathrm{U}^{11}$ & $\mathrm{U}^{22}$ & $\mathrm{U}^{33}$ & $U^{23}$ & $\mathrm{U}^{13}$ & $\mathrm{U}^{12}$ \\
\hline $\mathrm{N}(1)$ & $26(1)$ & $23(1)$ & $36(1)$ & $8(1)$ & $3(1)$ & $6(1)$ \\
\hline $\mathrm{N}(2)$ & $45(1)$ & $43(1)$ & $66(2)$ & $14(1)$ & $18(1)$ & $-4(1)$ \\
\hline $\mathrm{C}(1)$ & $26(1)$ & $25(1)$ & $24(1)$ & $1(1)$ & $-2(1)$ & $0(1)$ \\
\hline$C(2)$ & $27(1)$ & $28(1)$ & $36(1)$ & $8(1)$ & $0(1)$ & 2(1) \\
\hline $\mathrm{C}(4)$ & $30(1)$ & $31(1)$ & $29(1)$ & $5(1)$ & $2(1)$ & $-4(1)$ \\
\hline$C(3)$ & $23(1)$ & $32(1)$ & $40(2)$ & $4(1)$ & $1(1)$ & $1(1)$ \\
\hline$C(5)$ & $29(1)$ & $24(1)$ & $29(1)$ & $4(1)$ & $0(1)$ & $0(1)$ \\
\hline$C(6)$ & $28(1)$ & $23(1)$ & $22(1)$ & $2(1)$ & $0(1)$ & $-1(1)$ \\
\hline $\mathrm{C}(7)$ & $30(1)$ & $22(1)$ & $28(1)$ & $0(1)$ & $-2(1)$ & $4(1)$ \\
\hline$C(8)$ & $27(1)$ & $22(1)$ & $24(1)$ & $-4(1)$ & $-1(1)$ & $2(1)$ \\
\hline$C(9)$ & $26(1)$ & $34(1)$ & $36(1)$ & $2(1)$ & $5(1)$ & $3(1)$ \\
\hline$C(10)$ & $29(1)$ & $33(1)$ & $34(1)$ & 2(1) & $3(1)$ & $-4(1)$ \\
\hline $\mathrm{C}(11)$ & $30(1)$ & $26(1)$ & $28(1)$ & $0(1)$ & $2(1)$ & $-3(1)$ \\
\hline$C(12)$ & $24(1)$ & $25(1)$ & $26(1)$ & $1(1)$ & 1(1) & $0(1)$ \\
\hline $\mathrm{C}(13)$ & $32(1)$ & $33(1)$ & $45(2)$ & $3(1)$ & $6(1)$ & $-1(1)$ \\
\hline $\mathrm{C}(14)$ & $37(1)$ & $40(1)$ & $29(1)$ & $1(1)$ & $-6(1)$ & $-2(1)$ \\
\hline
\end{tabular}


Table S10. Hydrogen coordinates ( $\left.\times 10^{4}\right)$ and isotropic displacement parameters $\left(\AA^{2} \times 10^{3}\right)$ for $\mathbf{4 c}$.

\begin{tabular}{|c|c|c|c|c|}
\hline & $\mathrm{x}$ & $\mathrm{y}$ & $\mathrm{z}$ & $\mathrm{U}(\mathrm{eq})$ \\
\hline $\mathrm{H}(1)$ & $3950(14)$ & $6730(40)$ & $6090(20)$ & $31(6)$ \\
\hline $\mathrm{H}(2 \mathrm{~A})$ & 2736 & 6127 & 7001 & 36 \\
\hline $\mathrm{H}(3 \mathrm{~A})$ & 1963 & 3771 & 8167 & 38 \\
\hline $\mathrm{H}(5 \mathrm{~A})$ & 3672 & -839 & 8467 & 33 \\
\hline $\mathrm{H}(7 \mathrm{~A})$ & 4993 & 263 & 7720 & 32 \\
\hline $\mathrm{H}(9 \mathrm{~A})$ & 6161 & 1313 & 5847 & 38 \\
\hline $\mathrm{H}(9 \mathrm{~B})$ & 6298 & 2823 & 7060 & 38 \\
\hline $\mathrm{H}(10 \mathrm{~A})$ & 6622 & 5660 & 5752 & 38 \\
\hline $\mathrm{H}(10 \mathrm{~B})$ & 6212 & 4422 & 4614 & 38 \\
\hline $\mathrm{H}(11 \mathrm{~A})$ & 5503 & 7297 & 6430 & 34 \\
\hline $\mathrm{H}(11 \mathrm{~B})$ & 5381 & 7416 & 4970 & 34 \\
\hline $\mathrm{H}(14 \mathrm{D})$ & 4162 & 2647 & 4602 & 53 \\
\hline $\mathrm{H}(14 \mathrm{~A})$ & 5006 & 3024 & 4113 & 53 \\
\hline $\mathrm{H}(14 \mathrm{~B})$ & 4399 & 5049 & 4008 & 53 \\
\hline
\end{tabular}


Table S11. Examination of catalyst on the hydroamination of triene with aniline ${ }^{a}$

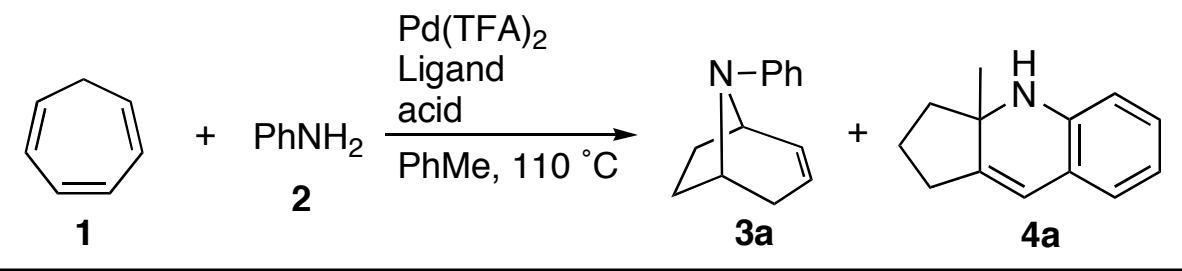

\begin{tabular}{|c|c|c|c|c|c|}
\hline entry & ligand & acid & time $(\mathrm{h})$ & yield: $\mathbf{3 \mathbf { a } ^ { b }}(\%)$ & $\mathbf{4} \mathbf{a}^{b}(\%)$ \\
\hline 1 & $t$ BuXantphos ${ }^{c}$ & - & 24 & 67 & 20 \\
\hline 2 & $t$ BuXantphos & $10 \% \mathrm{TfOH}$ & 15 & 12 & 25 \\
\hline 3 & $t$ BuXantphos & $10 \%$ TFA & 15 & 58 & 28 \\
\hline 4 & $t$ BuXantphos & $10 \% \mathrm{PhCO}_{2} \mathrm{H}$ & 2 & 78 & 20 \\
\hline 5 & Xantphos $^{d}$ & - & $<2$ & 81 & 17 \\
\hline 6 & Xantphos & $10 \% \mathrm{PhCO}_{2} \mathrm{H}$ & $<2$ & 82 & 14 \\
\hline 7 & DPEphos $^{e}$ & - & 15 & 66 & 14 \\
\hline 8 & DPFphos $^{f}$ & - & 15 & 20 & 8 \\
\hline 9 & $\mathrm{Ph}_{3} \mathrm{P}$ & - & 24 & 19 & 26 \\
\hline $10_{o}$ & $t \mathrm{Bu}_{3} \mathrm{P}$ & - & 24 & NR & NR \\
\hline $11^{g}$ & $\mathrm{DPPF}^{h}$ & - & 15 & $(37)^{i}$ & (46) \\
\hline
\end{tabular}

${ }^{a}$ Reaction conditions: triene $(2.0 \mathrm{mmol}, 4$ eq for 2$)$, aniline $(0.5 \mathrm{mmol}), \mathrm{Pd}(2 \%)$, ligand $(4 \%)$, acid $(10 \%)$, and toluene $(0.5 \mathrm{~mL}) .{ }^{b} \mathrm{GC}$ Yield. ${ }^{c}$ tBuXantphos $=2,7$-ditert-butyl-9,9-dimethyl-4,5-bis(diphenylphosphino)xanthene. $d$ Xantphos $=9,9$ dimethyl-4,5-bis(diphenylphosphino)xanthene. $e^{e}$ DPEphos $=$ Bis(2-diphenyl phosphinophenyl)ether. ${ }^{f}$ DPFphos $=4,5$-Bis (diphenylphosphino)dibenzofuran. ${ }^{g} 5 \%$ of $\mathrm{Pd}(\mathrm{TFA})_{2}$ and the ligand were used. ${ }^{h} \mathrm{DPPF}=1,1$ '-Bis(diphenylphosphino) ferrocene. ${ }^{i}$ Isolated yields are in parenthesis. 


\section{References}

(1) Supple, J. H.; Eklum, E. J. Am. Chem. Soc. 1971, 93, 6684. 